\title{
Archeops: A High Resolution, Large Sky Coverage Balloon Experiment for Mapping CMB Anisotropies
}

\author{
A. Benoît ${ }^{\mathrm{a}}$, P. Ade ${ }^{\mathrm{b}}$, A. Amblard ${ }^{\mathrm{c}}$, R. Ansari ${ }^{\mathrm{d}}$, \\ E. Aubourg ${ }^{\text {e }}$, J. Bartlett ${ }^{\mathrm{f}}$, J.-P. Bernard ${ }^{g}$, R. S. Bhatia ${ }^{\mathrm{h}}$, \\ A. Blanchard ${ }^{\text {f }}$, J. J. Bock ${ }^{\mathrm{i}}$, A. Boscaleri ${ }^{\mathrm{j}}$, F. R. Bouchet ${ }^{\mathrm{k}}$, \\ A. Bourrachot ${ }^{\mathrm{d}}$, P. Camus ${ }^{\ell}$, F. Couchot ${ }^{\mathrm{d}}$, P. de Bernardis ${ }^{\mathrm{m}}$, \\ J. Delabrouille ${ }^{\mathrm{c}}$, F.-X. Désert ${ }^{\mathrm{n}}$, O. Doré ${ }^{\mathrm{k}}$, M. Douspis ${ }^{\mathrm{f}}$, \\ L. Dumoulin ${ }^{\ell}$, X. Dupac ${ }^{\circ}$, P. Filliatre ${ }^{\mathrm{p}}$, K. Ganga ${ }^{\mathrm{c}}$, \\ F. Gannaway ${ }^{\mathrm{b}}$, B. Gautier ${ }^{\mathrm{a}}$, M. Giard $^{\mathrm{o}}$, \\ Y. Giraud-Héraud ${ }^{\text {c }}$, R. Gispert ${ }^{\dagger}$ g,1, L. Guglielmi ${ }^{\text {c }}$, \\ J.-C. Hamilton ${ }^{c}$, S. Hanany q, S. Henrot-Versillé ${ }^{\mathrm{d}}$, \\ V. V. Hristov ${ }^{\text {h, J. Kaplan }}{ }^{\text {c }}$, G. Lagache ${ }^{g}$, J.-M. Lamarre ${ }^{g}$, \\ A. E. Lange ${ }^{\mathrm{h}}$, K. Madet ${ }^{\mathrm{a}}$, B. Maffei ${ }^{\mathrm{b}}$, D. Marrone ${ }^{\mathrm{q}}$, \\ S. Masi ${ }^{\mathrm{m}}$, J. A. Murphy ${ }^{\mathrm{r}}$, F. Naraghi ${ }^{\mathrm{p}}$, F. Nati ${ }^{\mathrm{m}}$, \\ G. Perrin ${ }^{p}$, M. Piat ${ }^{g}$, J.-L. Puget ${ }^{g}$, D. Santos ${ }^{\mathrm{p}}$, \\ R. V. Sudiwala ${ }^{\mathrm{b}}$, J.-C. Vanel ${ }^{\mathrm{c}}$, D. Vibert ${ }^{\mathrm{k}}$, E. Wakui ${ }^{\mathrm{b}}$, \\ D. Yvon ${ }^{\mathrm{e}}$ \\ a Centre de Recherche sur les Très Basses Températures, 25 Avenue des Martyrs \\ BP166, F-38042 Grenoble Cedex 9, France \\ b Queen Mary and Westfield College, London, UK \\ c Physique Corpusculaire et Cosmologie, College de France, 11 pl. Marcelin \\ Berthelot, F-75231 Paris Cedex 5, France \\ d Laboratoire de l'Accélérateur Linéaire, BP 34, Campus Orsay, 91898 Orsay \\ Cedex, France \\ e SPP/DAPNIA/DSM, CEA-Saclay, F-91191 Gif-sur-Yvette Cedex, France \\ f Observatoire de Midi-Pyrénées, 14 Avenue E. Belin, 31400 Toulouse, France \\ g Institut d'Astrophysique Spatiale, Bât. 121, Université Paris XI, F-91405 Orsay \\ Cedex, France \\ ${ }^{\mathrm{h}}$ California Institute of Technology, Pasadena, CA, USA \\ i Jet Propulsion Laboratory, Pasadena, CA, USA \\ j IROE-CNR, Firenze, Italy \\ ${ }^{\mathrm{k}}$ Institut d'Astrophysique de Paris, 98bis, Boulevard Arago, 75014 Paris, France
}


${ }^{\ell}$ CSNSM-IN2P3, Bât 108, 91405 Orsay Campus, France

${ }^{\mathrm{m}}$ Gruppo di Cosmologia Sperimentale, Dipartimento di Fisica, Universita "La Sapienza", P. A. Moro, 2, 00185 Roma, Italy

${ }^{n}$ Laboratoire d'Astrophysique, Observatoire de Grenoble BP 53, 414 rue de la piscine, F-38041 Grenoble Cedex 9, France

- Centre d'Étude Spatiale des Rayonnements, 9 avenue du Colonel Roche, BP 4346, F-31028 Toulouse Cedex 4, France

p Institut des Sciences Nucléaires, 53 Avenue des Martyrs, 38026 Grenoble Cedex, France

${ }^{q}$ School of Physics and Astronomy, 116 Church St. S.E., University of Minnesota, Minneapolis MN 55455, USA

${ }^{\mathrm{r}}$ Experimental Physics, National University of Ireland, Maynooth, Ireland

\begin{abstract}
Archeops is a balloon-borne instrument dedicated to measuring cosmic microwave background $(\mathrm{CMB})$ temperature anisotropies at high angular resolution $(\sim 8$ arcminutes) over a large fraction $(\sim 25 \%)$ of the sky in the millimetre domain. Based on Planck High Frequency Instrument (HFI) technology, cooled bolometers (0.1 K) scan the sky in total power mode with large circles at constant elevation. During the course of a 24-hour Arctic-night balloon flight, Archeops will observe a complete annulus on the sky in four frequency bands centered at 143, 217, 353 and $545 \mathrm{GHz}$ with an expected sensitivity to CMB fluctuations of $\sim 100 \mu \mathrm{K}$ for each of the 90 thousand 20 arcminute average pixels. We describe the instrument and its performance obtained during a test flight from Trapani (Sicily) to Spain in July 1999.
\end{abstract}

Key words:

Cosmology, 98.80;

Cosmic dust Milky Way, 98.38;

Detectors bolometers, 07.57.K;

Instruments for astronomy, 95.55;

$\overline{1}$ Richard Gispert passed away few weeks after his return from the mission to Trapani 


\section{Introduction}

The anisotropies of the cosmic microwave background (CMB) are a goldmine of cosmological information. They tell us about the state of the Universe at the tender age of a few hundred thousand years (redshift $\sim 1000$ ) when the primeval plasma recombined and unveiled the small fluctuations that would eventually form the large-scale structure of the present-day Universe. The applicability of linear perturbation theory to these small inhomogeneities provides the cosmologist with a relatively direct link between observable and theoretical quantities over a large range of physical scales. Accurate measurements of the anisotropies can therefore be used to constrain fundamental parameters of the standard Big Bang cosmogoly, at least within the adopted theoretical framework (e.g., Inflation). For the case of inflation-generated perturbations, this approach most directly constrains the spatial geometry of the Universe, the physical particle densities (such as the baryon or dark matter density) and the form of the primeval perturbation spectrum (scalar and tensor modes), as well as the ionization history since recombination. A large international effort has set as its goal high precision measurements of the CMB anisotropies, employing numerous ground-based, balloon and space-borne projects; among the latter MAP to be launched by NASA in 2001, and Planck to be launched in 2007 by ESA.

Archeops is a balloon-borne instrument based on Planck High Frequency Instrument (HFI) technology that is optimized to cover a large fraction of sky at high angular resolution. The use of cold bolometers at the focus of a telescope with an aperture of 1.3 meter allows the HFI to reach an angular resolution better than 10 arcmin at millimeter wavelengths. The goal for Archeops is to map $\sim 25 \%$ of the sky with 8 arcmin resolution in four frequency bands - 143, 217, 353 and $545 \mathrm{GHz}$ - to obtain a limiting CMB sensitivity of $\Delta T / T \simeq 3 \times 10^{-5}$ per coadded 20 arcmin sky pixel. This is achieved by spinning the payload $(\sim 2-3 \mathrm{rpm})$ so that the detectors scan the sky at a constant elevation of $\sim 41^{\circ}$, mapping out a large annulus on the sky during a 24-hour flight. The angular resolution is achieved with a 1.5 meter aperture telescope, and sensitivity is obtained with 8 bolometers at $143 \mathrm{GHz}$, 8 at $217 \mathrm{GHz}, 6$ at $353 \mathrm{GHz}$, and 2 at $545 \mathrm{GHz}$. The CMB signal is recorded by the 143 and $217 \mathrm{GHz}$ detectors, and the 353 and $545 \mathrm{GHz}$ detectors monitor atmospheric and interstellar dust emission. A 24-hour night-time balloon flight is possible during the Arctic Winter from the Swedish Esrange Station (near Kiruna on the Arctic Circle) operated by the French Centre National d'Etudes Spatiales (CNES). Comparison in Fig. 1 with the current observations [Bennett et al, 1996], [de Bernardis et al., 2000], [Hanany et al., 2000] shows the potential of this type of mission and in particular its ability to probe a broad range of angular scales. Namely, it should not only give new measurements with an angular resolution high enough to probe the second peak an- 
gular domain, but its measurement of large angular scales fluctuations should also allow an absolute measurement of the height of the first peak. Indeed, by connecting for the first time in a single experiment large scale fluctuations as measured by COBE/DMR to contemporary medium scales measurements, it should offer an absolute calibration of the first "acoustic" peak height, an issue still unclear even after the BOOMERanG and MAXIMA results. Note that both these issues should be confirmed too by the TOPHAT balloon experiment [Martin et al., 1996] and MAP satellite. Timescale for Archeops experiment is to have two campaigns in the Winter 2000 and Winter 2001. Additional information and updates may be found at our web-site ${ }^{2}$.

In this paper we describe in detail the instrument and its performance during its test flight from the Italian Space Agency's base in Trapani (Sicily) in July 1999. The next section presents the instrument itself. This is followed in Section 3 by a discussion of the ground calibration befo re flight. General aspects of the test flight are given in Section 4, followed by a description of the in-flight performance (Sec. 5) and calibration (Sec. 6). Section 7 gives a brief conclusion.

\section{Hardware Description}

In this section we describe in detail the Archeops optics, detectors, electronics and flight package, including a star sensor for pointing reconstruction.

\subsection{Telescope}

The Archeops telescope is a two mirror, off-axis, tilted Gregorian telescope consisting of a parabolic primary and an elliptical secondary. An off-axis Gregorian system has higher aperture efficiency and lower sidelobe response compared to an equivalent f-number on-axis system [Dragone \& Hogg, 1974]. The telescope satisfies the Mizuguchi-Dragone condition [Mizuguchi, Akagawa \& Yokoi, 1978], [Dragone, 1982] in which astigmatism is canceled to first order and there is no cross-polarization in the center of the field of view. Cross-polarization is also small away from the center of the field.

The telescope was designed to provide diffraction-limited performance when coupled to single mode horns producing beams with FWHM of 8 arcminutes or less at frequencies higher than $\sim 140 \mathrm{GHz}$. The target beam sizes, wavelengths and edge taper are similar to those designed for the HFI on board the Planck

$\overline{2 \text { http }}$ //Www.archeops.org 

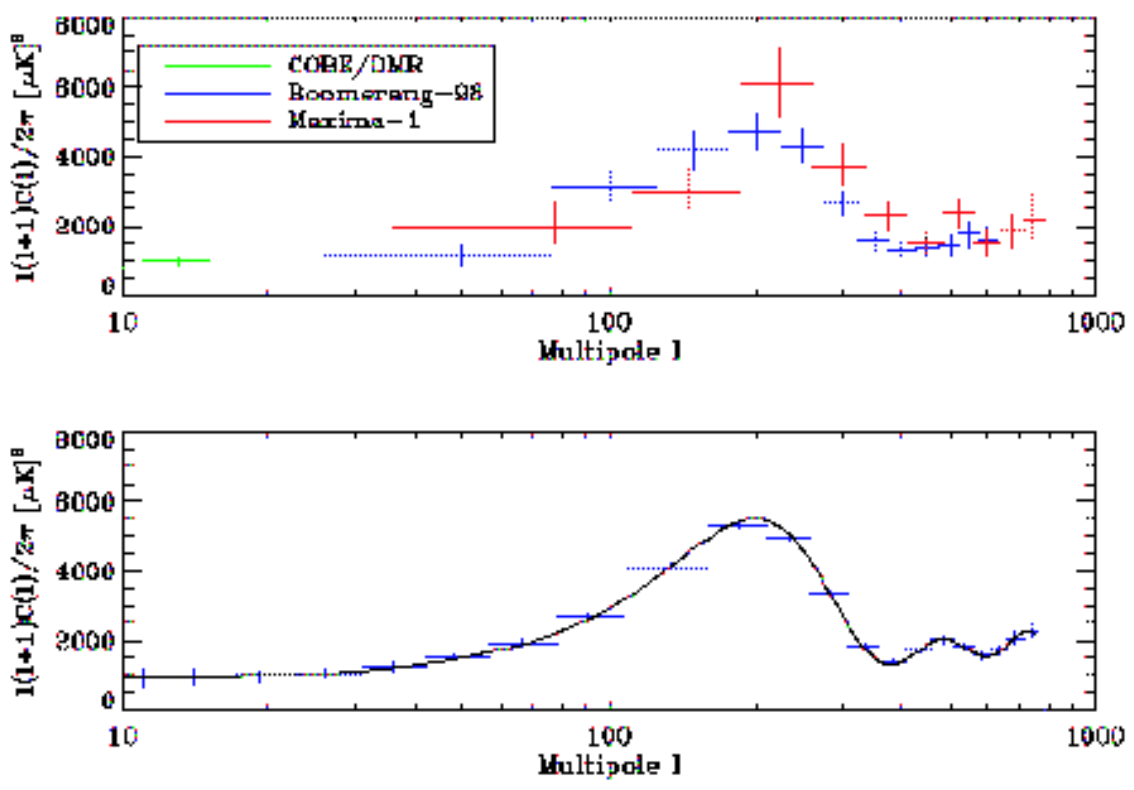

Fig. 1. The expected accuracy with which the CMB temperature power spectrum could be determined using a 24-hour data from Archeops. Top: COBE/DMR, B98 and Maxima-1 independent band powers [Bennett et al, 1996], [de Bernardis et al., 2000], [Hanany et al., 2000]. Bottom: the COBE/DMR, B98 and Maxima-1 joint analysis best fit with weak prior (solid line) [Jaffe et al., 2000] and expected band powers from Archeops (data points) in bins that have a constant $\Delta l n \ell=0.375$ below $\ell=107$ and a constant $\Delta \ell=50$ at higher $\ell$ 's. A 24-hour Archeops data set should provide an important overlap between COBE data and current higher resolution measurements, thus giving a strong determination of the absolute amplitude of the first acoustic peak. The expected error bars do not include systematic and calibration uncertainties or the effects of removing foregrounds. We 
satellite ${ }^{3}$. Within a circular radius of $1.8^{\circ}$ from the center of the field-of-view, the telescope has a wave-front error of less than $6 \%$ at all wavelengths.

Table 1 lists the specifications of the telescope and a schematic is shown in Figs. 2 and 3. The primary mirror is the aperture stop of the system and is defined as the intersection of a cylinder of 1.5 meter diameter with an off-axis section of a paraboloid. It has an elliptical shape with a length of 1.8 meters and 1.5 meters for the major and minor axes, respectively. The oversized secondary is close to circular, with a diameter of about $0.8 \mathrm{~m}$.

Both mirrors were milled from 8 inch thick billets of aluminum 6061-T6 and were thermally cycled twice during machining to relieve internal stresses. The $5 \mathrm{~mm}$ thick reflecting surfaces are supported by a honeycomb rib structure that reduces deflections due to gravity and milling machine pressure (during machining), and which ensures that the material does not exceed its yield strength under the expected load of $10 \mathrm{~g}$ during parachute shock at the end of the flight. The primary mirror construction is shown in Fig. 3. The primary and secondary mirrors weigh $45 \mathrm{~kg}$ and $10 \mathrm{~kg}$, respectively. The mirrors were hand polished to provide the required figure accuracy. The local surface roughness is less than $2 \mu \mathrm{m}$ roughness average [Green, 1996], and the overall figure accuracy is $50 \mu \mathrm{m}$ rms.

\subsection{Focal plane}

The focal plane configuration during the flight in Trapani consisted of a total of six photometric pixels, three at the $143 \mathrm{GHz}$ band (B1-1,2,3 $\equiv$ Band 1; pixels $1,2,3)$, two at the $217 \mathrm{GHz}$ band (B2-4,5) and one at the $353 \mathrm{GHz}$ band (B3-6). A photometric pixel is defined as the assembly of one bolometer and its front cold optics (see Fig. 4). The angular separation between the photometric pixels in the focal plane was roughly 50 arcmin. Fig. 21 shows an inflight measurement of the focal plane geometry (see section 5.2.3). For Kiruna flights, it is anticipated that the angular separation between nearby bolometers will be 30 arcmin.

\subsubsection{Photometric pixel layout}

The configuration of the focal plane is similar to that being developed for Planck HFI. For CMB anisotropy measurements, control of spectral leaks and beam sidelobe response is critical. Archeops channels have been specifically

3 Thus, the optical design is similar to the one proposed for Planck during phase-A of the project except that the Archeops telescope has lower edge taper, because of the significantly larger primary mirror, and better optical performance. 


\begin{tabular}{l|l}
\hline \multicolumn{1}{c|}{ Parameter } & Value \\
\hline $\mathrm{f \#}^{a}$ & 1.7 \\
Plate Scale $^{a}$ & $35 \mathrm{~mm} / \mathrm{deg}$ \\
\hline Primary Mirror & \\
$\quad$ conic constant & -1 \\
radius of curvature of paraboloid & $160 \mathrm{~cm}$ \\
major axis of mirror & $177 \mathrm{~cm}$ \\
minor axis of mirror & $150 \mathrm{~cm}$ \\
weight & $45 \mathrm{~kg}$ \\
\hline Secondary Mirror & \\
conic constant & -0.18 \\
radius of curvature & $53 \mathrm{~cm}$ \\
tilt of major axis ${ }^{a}$ & $15 \mathrm{degrees}$ \\
major axis of ellipsoid & $130 \mathrm{~cm}$ \\
axial magnification of elliposoid \\
major axis of mirror \\
minor axis or mirror \\
weight
\end{tabular}

Table 1

${ }^{a}$ Value at the center of the focal plane

Design and construction parameters for the Archeops telescope.

designed to maximize the sensitivity to the desired signal, while rejecting outof-band or out-of-beam radiation. Developing on a design put forward for a previous proposed mission [Church et al., 1996], we have chosen to use a triple horn configuration for each photometric pixel, as shown schematically in Fig. 4. In this scheme, radiation from the telescope is focussed into the entrance of a back-to-back horn pair. With no optical components in the path, control of the beam is close to ideal, as will be shown later. A lens at the exit aperture of the second horn creates a beam-waist where wavelength selective filters can be placed. Finally, a second lens on the front of the third horn maintains beam control and focuses the radiation onto the spider bolometer placed at the exit aperture. A convenient aspect of this arrangement is that the various 


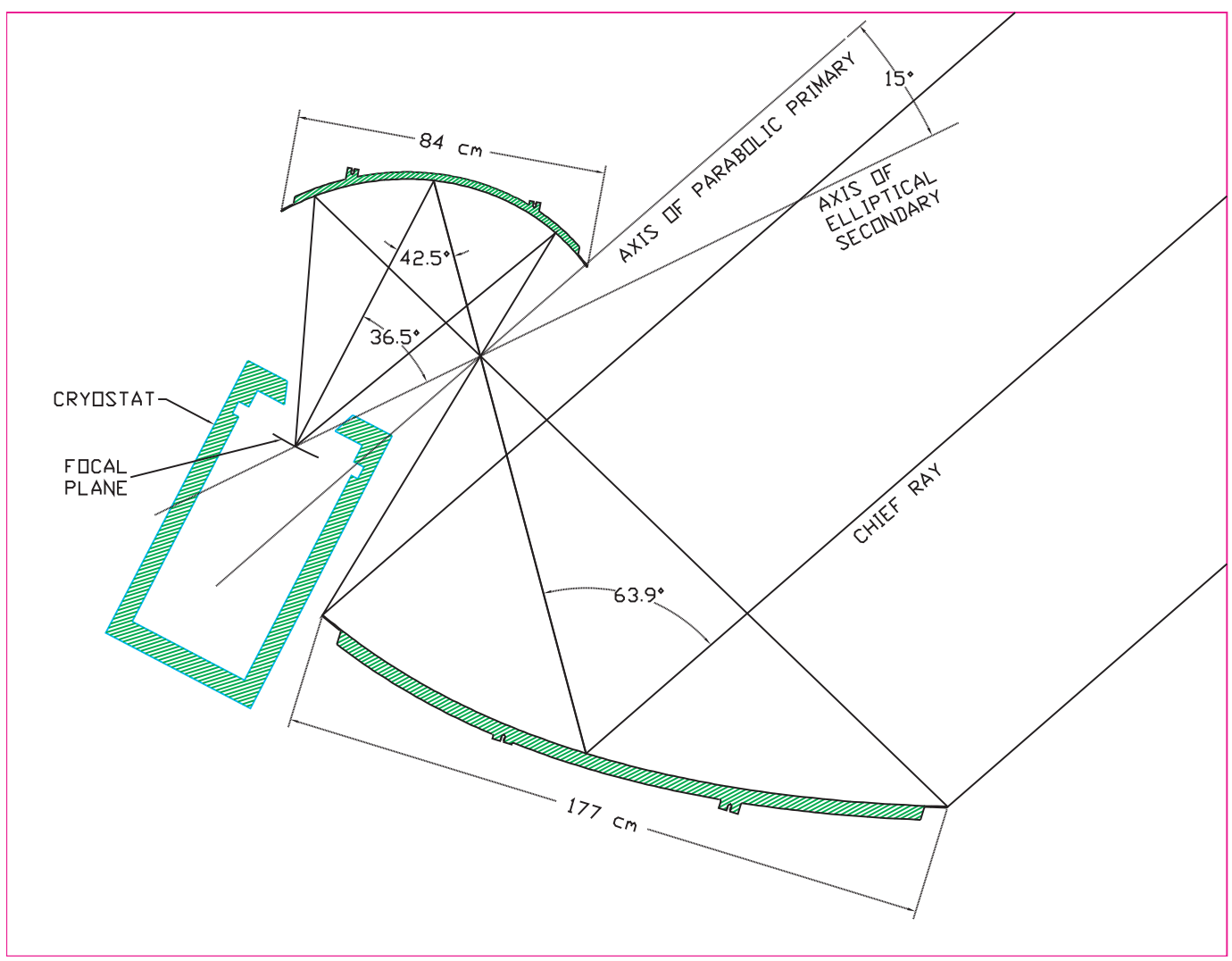

Fig. 2. A side view of the Archeops telescope and cryostat. The telescope is a tilted Gregorian system consisting of a parabolic primary and elliptic secondary. The primary axis of the ellipse is tilted with respect to the axis of the parabola such that the system satisfies the Dragone condition. The system provides an RMS wave-front error of less than $6 \%$ at wavelengths between 2 and $0.85 \mathrm{~mm}$, for beam sizes between 8 and 5 arcminute, respectively.

components can be placed on different temperature stages in order to create thermal breaks and to reduce the level of background power falling onto the bolometer and fridge.

In Archeops, the back-to-back horn pair is located on a cold plate cooled by Helium vapor. In flight it reaches a temperature of about $10 \mathrm{~K}$ because of the optical load from the telescope and the atmosphere. This results in some power loading on the detectors from the horns. A model of the photometric performance of the system shows that even with this high horn temperature, the detector is limited by the power radiated by the telescope and atmosphere, which determine the fundamental limit for this sub-orbital experiment. The back-to-back horn pair is constructed from two horns separated by a waveguide section. Sidelobe response, beamwidth on the sky and spillover are accurately controled by the design of the front horn. The waveguide is critical in that it defines the spectral high-pass cut-on for the band and controls the allowable modes that propagate from the telescope to the detector. Low pass filters placed at the exit of the horn pair reject all high frequency radiation 

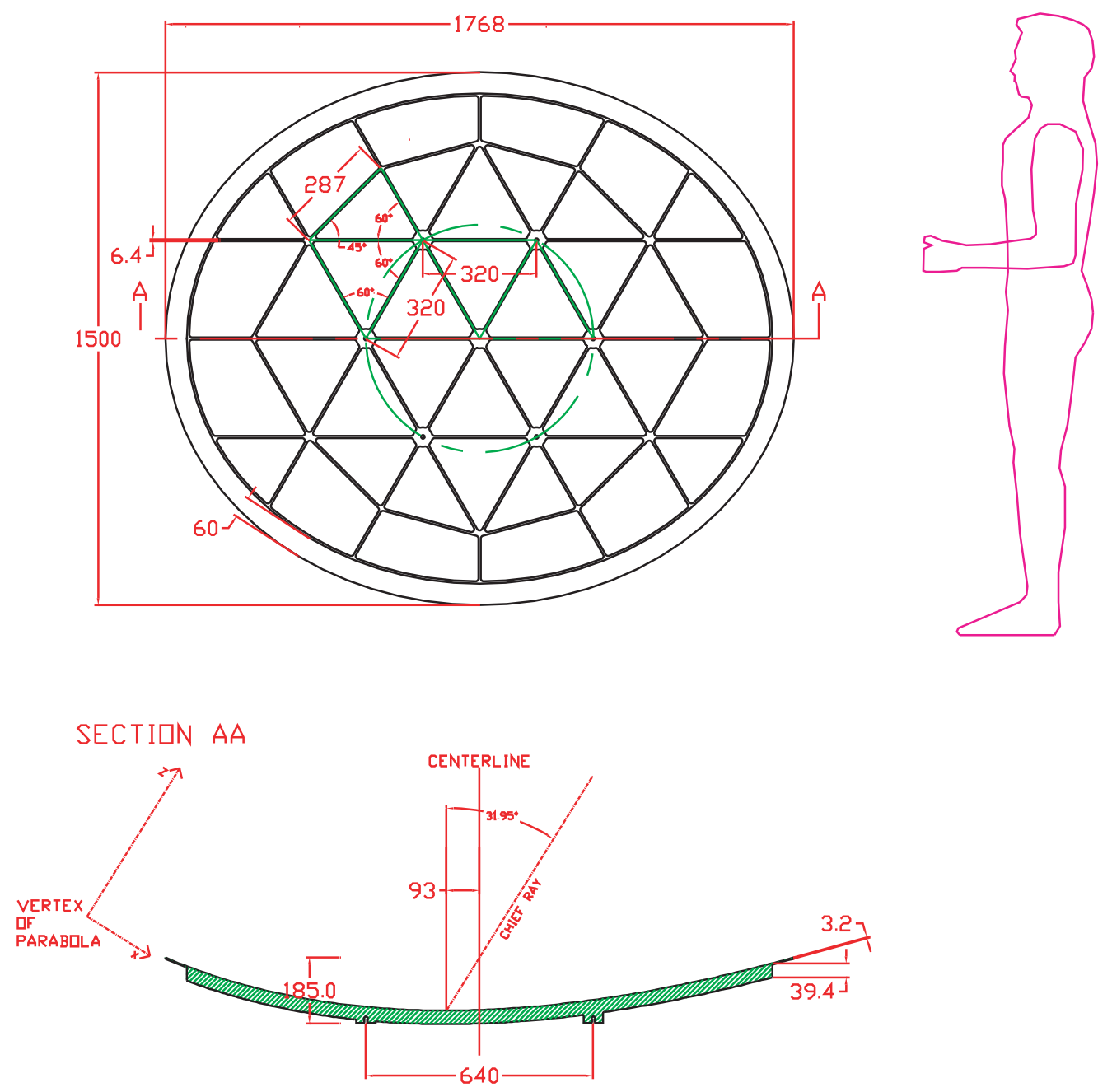

Fig. 3. A side and back view of the Archeops telescope primary mirror.

from the inner stages and bolometer.

To further shield the dilution refrigerator from unwanted thermal power, it is surrounded by a shield at $1.6 \mathrm{~K}$. With our optics scheme we were able to place more low pass filters at this point to further reject unwanted radiation from the inner sanctum where the $100 \mathrm{mK}$ detectors are located.

A second lens in front of the final $100 \mathrm{mK}$ horn refocuses the beam, which is then coupled to the bolometric detector by the horn and a further waveguide section. The diameter of this waveguide is set to be larger than the first backto-back waveguide to ensure that it does not modify the mode propagation of the feed system. The low pass band edge-defining filter was also located on the front of this horn at $100 \mathrm{mK}$.

For the Trapani test flight, all the 143 and $217 \mathrm{GHz}$ photometric pixels used 


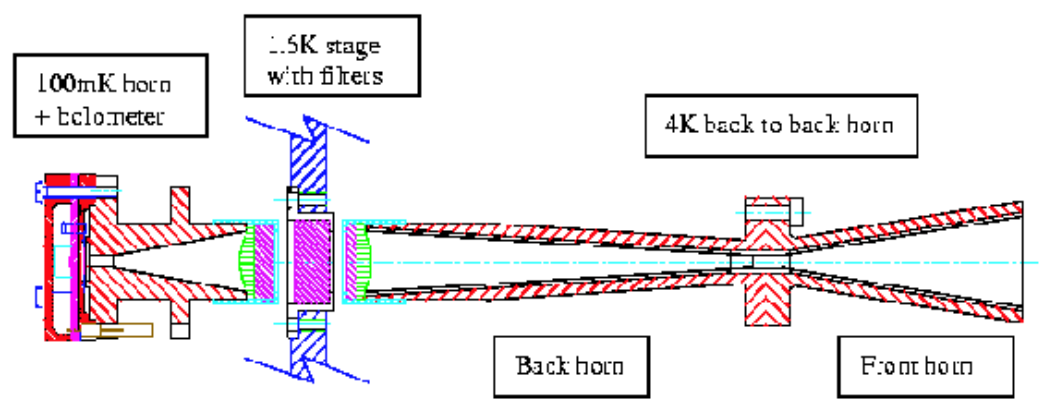

Fig. 4. Optical configuration for a single photometric pixel

identical conical corrugated back-to-back horns, which were single moded at $143 \mathrm{GHz}$ but allowed a few modes to propagate at $217 \mathrm{GHz}$. The $353 \mathrm{GHz}$ photometric pixel used a multi-moded smooth walled back-to-back horn. Measurements and models have shown [Maffei et al., 2000] that corrugation in the horns produce noticeable improvement in the symmetry of the antennal profiles between the $\mathrm{E}$ and $\mathrm{H}$ polarization patterns (see Fig. 5), and in the sidelobe levels. The use of the smooth walled horn for $353 \mathrm{GHz}$ was necessary because the only corrugated horns available were those designed for $143 \mathrm{GHz}$, which have poor transmission at these much higher frequencies [Colgan et al., 2000]. Proper single mode corrugated feeds will be made for the Kiruna flights. Fig. 6 shows the measured antenna response for the corrugated horns that were used during the Trapani flight. We estimate that we have achieved $-25 \mathrm{~dB}$ telescope edge taper with the telescope/horns combination. New profiled-flared horns that will be used in the Kiruna flights will achieve $-30 \mathrm{~dB}$.

\subsubsection{Spectral Filtering}

To obtain a well-defined band edge and ensure high rejection for UV-opticalIR wavelengths, a series of metal mesh low-pass interference filters were used. Each filter was manufactured from several layers of photolithographically etched copper-patterns on polypropylene substrates and then hot pressed together to form a rugged filter [Lee et al., 1996]. The back-to-back horn waveg- 


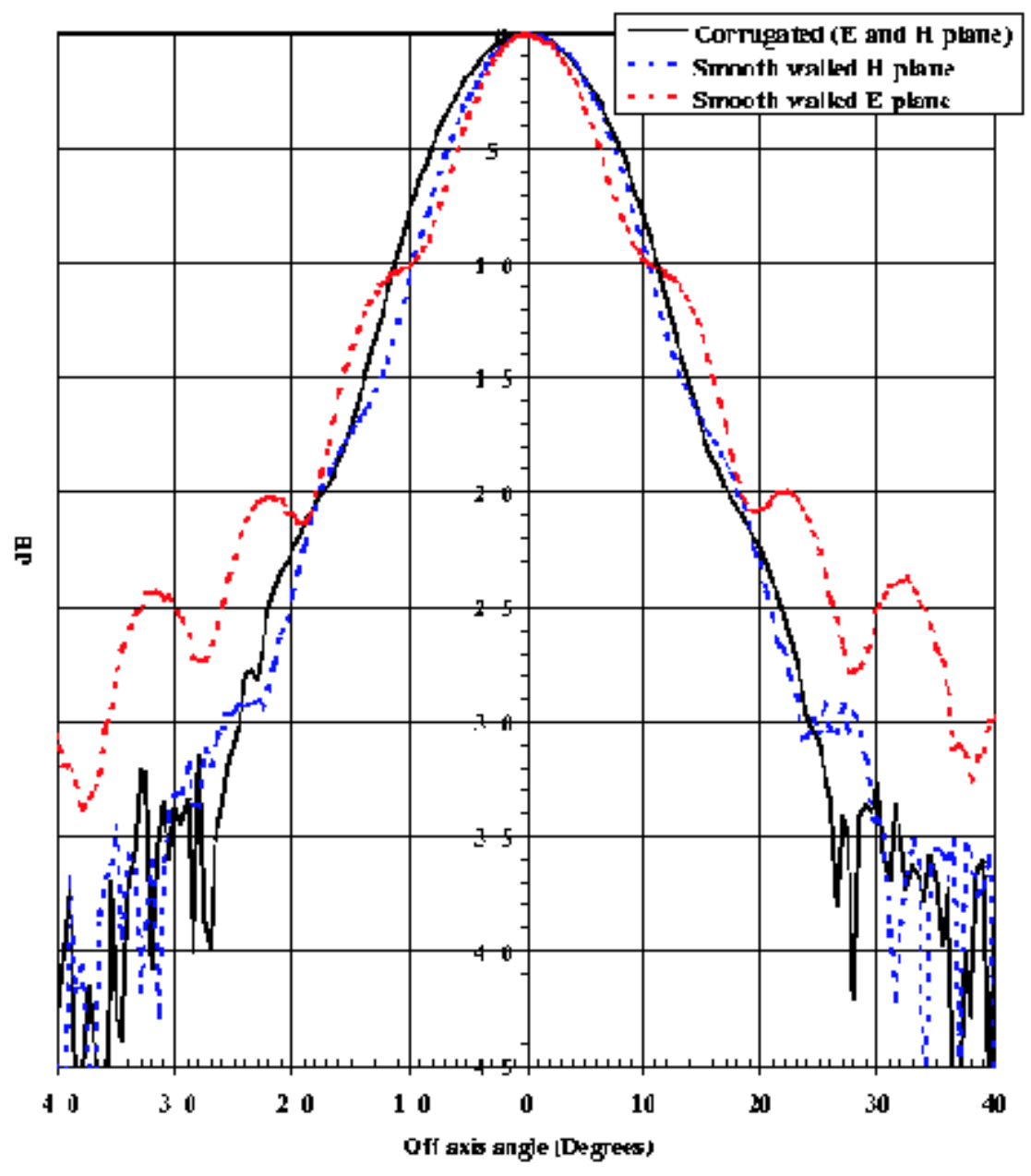

Fig. 5. Comparison of beam patterns ( $\mathrm{E}$ and $\mathrm{H}$ plane polarisation) measured at $150 \mathrm{GHz}$ for corrugated and smooth walled back to back horns. This figure shows the asymmetry between the 2 polarisations in the case of a smooth walled horn which will increase the sidelobe level, while in the case of a corrugated horn, the 2 polarisations are identical (only one is shown) down to the level of $35 \mathrm{~dB}$ (measurement limit). 


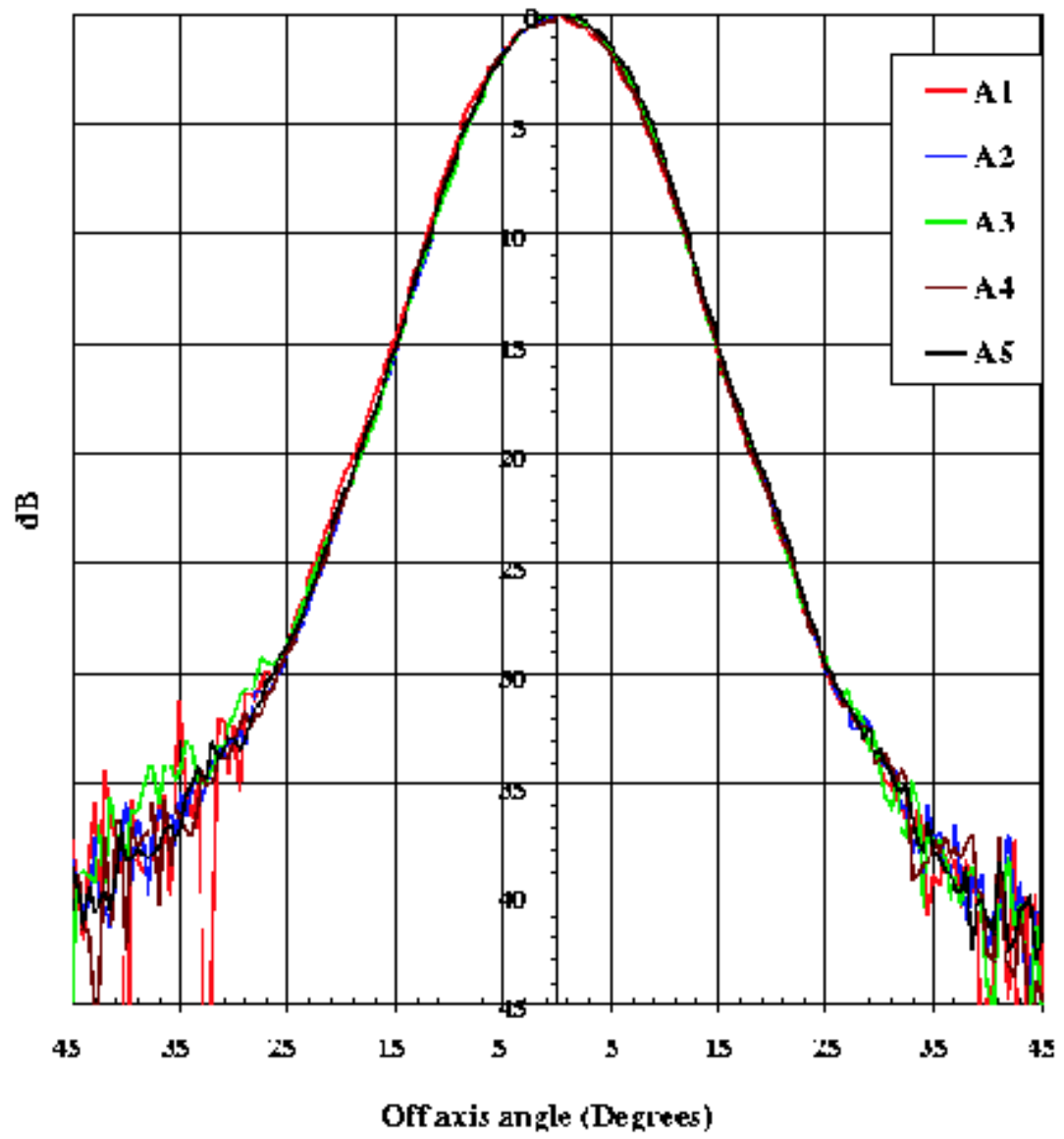

Fig. 6. Measured beam patterns at $180 \mathrm{GHz}$ for all the corrugated horns used during the 1st flight of Archeops in Trapani. We can notice the good mechanical reproducibility in the horn manufacture which gives identical beams for all the horns. 


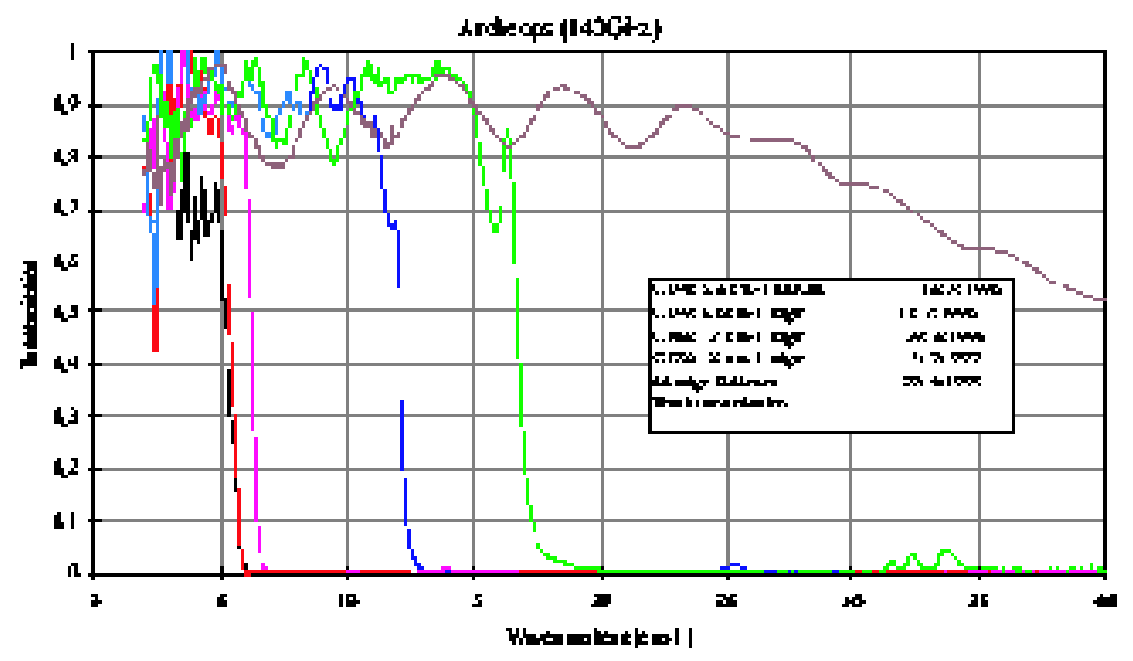

Fig. 7. Transmission of all filter components for the $143 \mathrm{GHz}$ channel. Total transmission is in black

uide defines the low frequency cut-on, and hence by choice of filters, the number of waveguide modes propagated. Fig. 7 shows the measured response on a linear plot for all the filter components used in the $143 \mathrm{GHz}$ channel. Outof-band rejection is better by a factor $10^{-10}$ in the submillimetre domain. Tests on similar filter stacks for Planck HFI show that the good rejection is maintained through the near IR, optical and UV regions. It is clear that the total in-band spectral transmission is typically 55\%. The complete expected transmission of the cold optics filtering scheme is shown in Fig. 8.

\subsubsection{Bolometric detectors}

The spider web bolometric detectors were developed by JPL/Caltech as part of the Planck HFI development. These bolometers have high responsivity, low NEP, fast speed of response and a low cross section to cosmic ray hits. The bolometers are made by micro-machining silicon nitride to leave a selfsupported, metallized spider web mesh absorber. A neutron-transmutationdoped Ge:Ga thermistor is then indium bump bonded at the web center to sense the temperature rise which results from the absorption of IR power [Bock et al., 1996], [Mauskopf et al., 1997]. The specific requirement for the Archeops detectors, which are cooled to $100 \mathrm{mK}$, is that the sensitivity should meet with the expected background limited photon noise $N E P_{B L I P}=1.5 \times$ $10^{-17} \mathrm{~W} / \mathrm{Hz}^{-1 / 2}$ and have a response time of $1.6 \mathrm{~ms}$, dictated by the instrument spin rate.

In preparation for the Trapani flight, three of the bolometers (photometric pixels B1-1, B1-3 and B3-6) were characterised as a function of operating temperature around $100 \mathrm{mK}$ using an adiabatic demagnetization refrigeration (ADR). Photometric pixel B1-1 was designed and used in Band 1, 143 GHz. Bolome- 


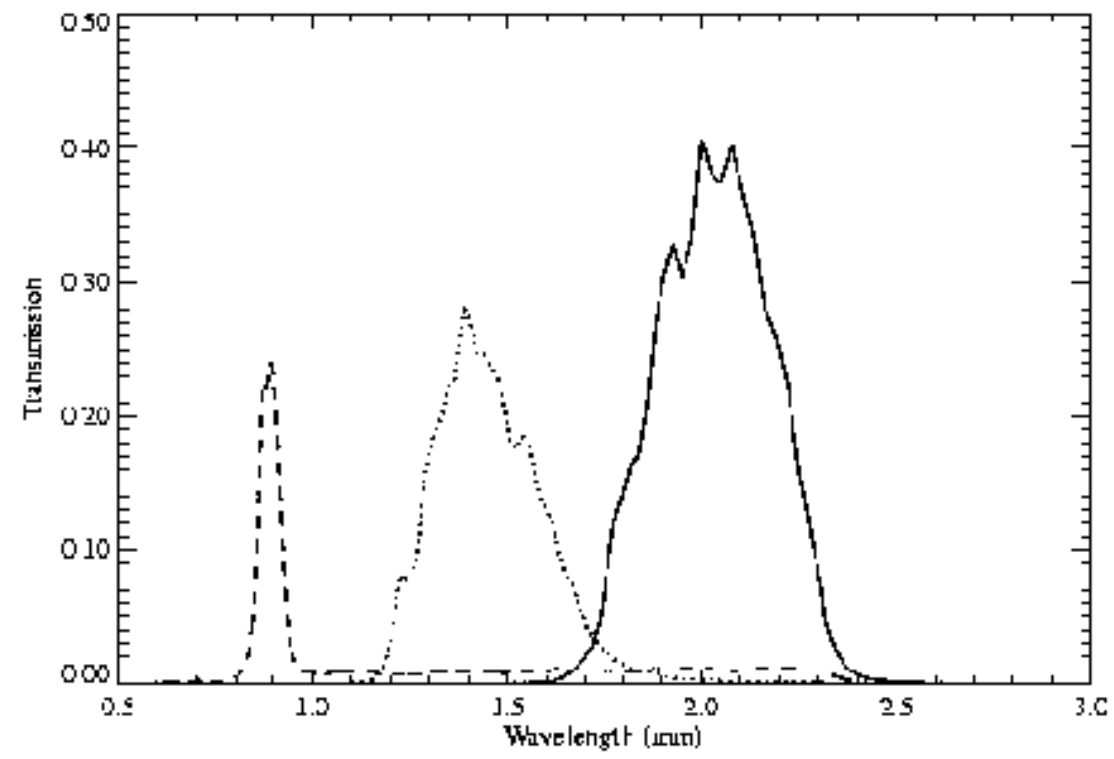

Fig. 8. Total transmission of Archeops channels obtained by combining separate measurements of each filtering components $(10 \mathrm{~K}, 1.6 \mathrm{~K}, 0.1 \mathrm{~K})$. Channel 1 (nominally taken, for calibration purpose, at $143 \mathrm{GHz}$ ) is centered at $150 \mathrm{GHz}$ roughly covering 134 to $164 \mathrm{GHz}$ at half maximum so that the bandpass is $\Delta \nu / \nu=0.20$. Channel 2 (nominally at $217 \mathrm{GHz}$ ) is centered at $211 \mathrm{GHz}$ roughly covering 188 to $236 \mathrm{GHz}$ at half maximum so that the bandpass is $\Delta \nu / \nu=0.23$. Channel 3 (nominally at $353 \mathrm{GHz}$ ) is centered at $341 \mathrm{GHz}$ roughly covering 329 to $354 \mathrm{GHz}$ at half maximum so that the bandpass is $\Delta \nu / \nu=0.074$. Due to lack of time before launch, this latter bandpass could not be optimised. The measurements are not accurate for wavelengths larger than about 2.5 mpq. 


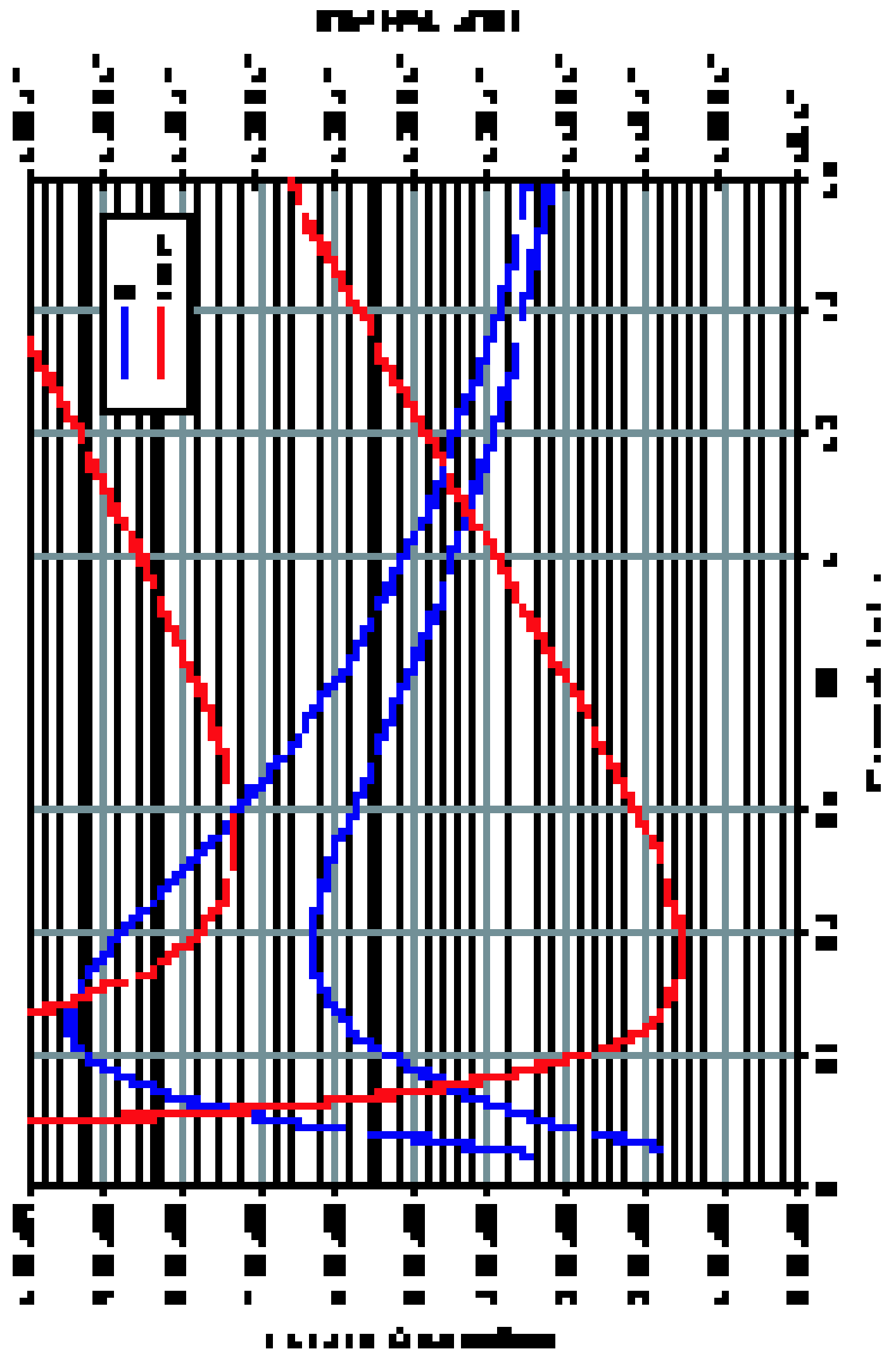

Fig. 9. The NEP and Responsivity versus bias current for pixels B1-1 and B3-6 at a base temperature of $98 \mathrm{mK}$. The bolometers are optically loaded with radiation from a $300 \mathrm{~K}$ blackbody attenuated to levels expected in flight. The NEP includes Johnson and phonon noise contributions. The optimum bias current, $I_{o p t}$, is when the $N E P$ is smallest. 
Table 2

Summary of the measured optimum bias current $I_{o p t}$, and the values of $N E P$, Responsivity (Resp) and optical time response $(\tau)$ of some bolometers operating at this bias current and a base temperature of $98 \mathrm{mK}$. The numbers are accurate to about $10 \%$.

\begin{tabular}{cllll} 
Bolometer & $\begin{array}{l}I_{\text {opt }} \\
(\mathrm{nA})\end{array}$ & $\begin{array}{l}10^{17} \times N E P \\
\left(\mathrm{WHz}^{-1 / 2}\right)\end{array}$ & $\begin{array}{l}\text { Resp } \\
(\mathrm{MV} / \mathrm{W})\end{array}$ & $\begin{array}{l}\tau \\
(\mathrm{ms})\end{array}$ \\
\hline B1-1 & 0.35 & 1.07 & 920 & 3.5 \\
B1-3 & 0.32 & 1.10 & 920 & 1.0 \\
B3-6 & 0.55 & 1.40 & 630 & 3.0 \\
\hline
\end{tabular}

ter 3-6 was designed for use in Band 2, $217 \mathrm{GHz}$, but was used in Band 3 at $353 \mathrm{GHz}$. Photometric pixel 3 uses a prototype Planck HFI $143 \mathrm{GHz}$ bolometer which was used in Band 1, $143 \mathrm{GHz}$. These three detectors were characterised optically in the ADR test-bed using prototype horns and filters optimised for $143 \mathrm{GHz}$ operation [Woodcraft et al. , 2000] and [Sudiwala et al. , 2000a]. The NEP and responsivity versus bias current for photometric pixels B1-1 and B3-6 operating at a base temperature of $98 \mathrm{mK}$ and under flight simulated optical loading conditions are shown in Fig. 9. The superimposed model fit curves have been calculated from the measured bolometer parameters using methods described by [Sudiwala et al. , 2000b]. These data, together with the measured optical response times, are summarised in Tab. 2.

\subsection{Cryostat}

The focal plane is cooled to $100 \mathrm{mK}$ by means of an open cycle dilution refrigerator designed at the CRTBT (Centre de Recherche sur les Très Basses Températures) in Grenoble (France) by [Benoît et al (1994a)] (see also [Benoît et al, 1994b]). The dilution stage is placed in a low temperature box placed on a liquid Helium reservoir at $4.2 \mathrm{~K}$. The top part of this box contains the entrance horns and receives a significant amount of heat power from near infrared radiation $(300-700 \mathrm{~mW})$. We therefore put in place a heat exchanger using exhaust vapours from the helium tank to maintain the horns near 10K (Fig. 10 and 11). The entrance is protected from radiation by two vapour cooled screens with openings for the input beam. The filters are placed on the horns at $10 \mathrm{~K}$, on the $1.6 \mathrm{~K}$ stage (cooled by Joule-Thompson expansion of the dilution mixture) and on the $100 \mathrm{mK}$ stage. The temperatures of each stage are monitored with thermometers: carbon resistance and $\mathrm{NbSi}$ metal insulation transition thermometers (see Sect. 2.3.2).

The bolometers are placed on the $100 \mathrm{mK}$ stage supported by Kevlar cords. The dilution fluids arrive through two small capillary tubes, after mixing, 


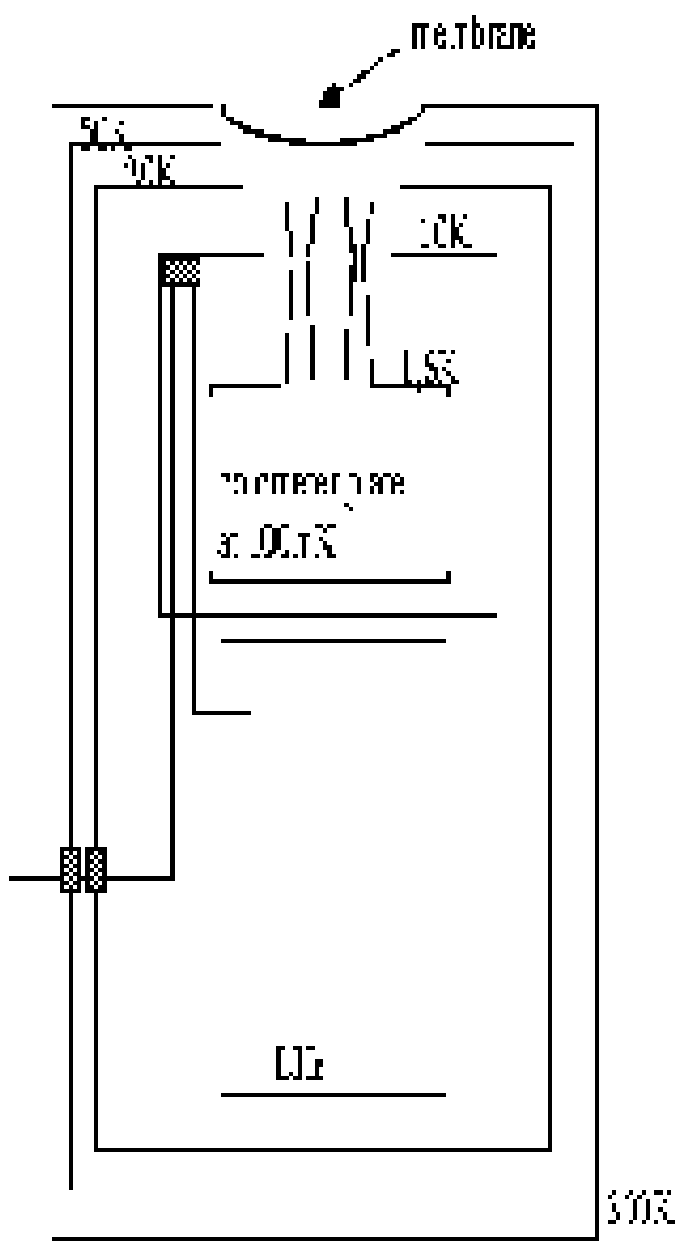




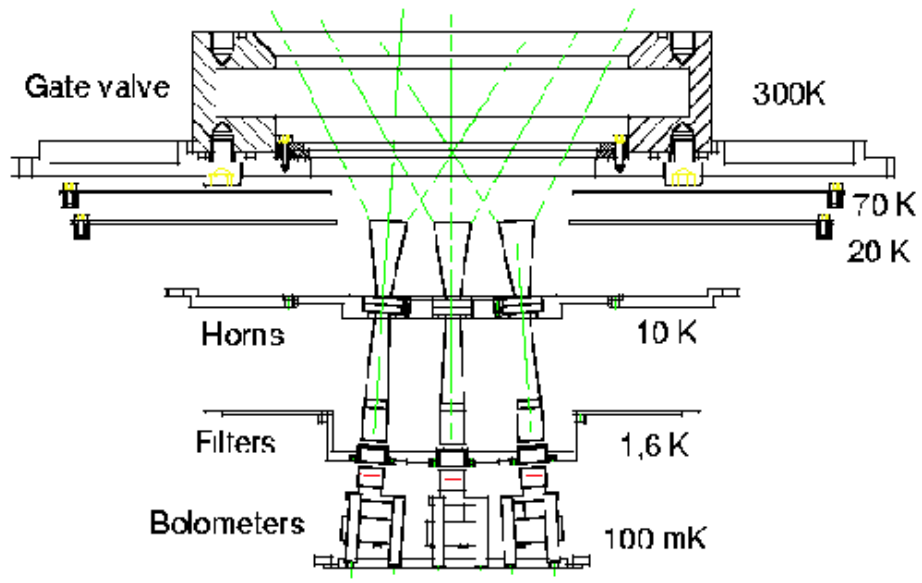

Fig. 11. Cryogenic and mechanical implementation of the cold optics in the cryostat.

and cool the stage using a small heat exchanger, finally exiting through a third capillary. These three capillary tubes, two extra capillary tubes used for precooling and the electric wires (9 shielded cables with 12 conductors each) are soldered together, forming the continuous heat exchanger disposed around the $100 \mathrm{mK}$ stage. The Planck HFI instrument design is similar. Input flow is controlled by an electronic flow regulator; the output mixture is pumped with a charcoal pump placed inside the liquid helium (1 liter box filled with charcoal). An electronic regulator is used to maintain constant pressure at one atmosphere in the helium tank.

\subsubsection{Thermal fluctuation control}

At the junction of the ${ }^{4} \mathrm{He}$ and ${ }^{3} \mathrm{He}$ tubes, a random sequence of concentrated and diluted ${ }^{3} \mathrm{He}$ phases is created, causing a temperature fluctuation of about $100 \mu \mathrm{K}$ and producing noise in the bolometers. The bolometer plate is connected to the $100 \mathrm{mK}$ dilution stage through a link with controlled thermal conduction (Fig. 12). An intermediate stage along this link can be used to regulate the temperature.

The first thermal link is composed of a vespel support and a brass link in parallel in order to regulate thermal conduction. Combined with a copper mass, the time constant of the intermediate stage is about 1 second. The main fil- 


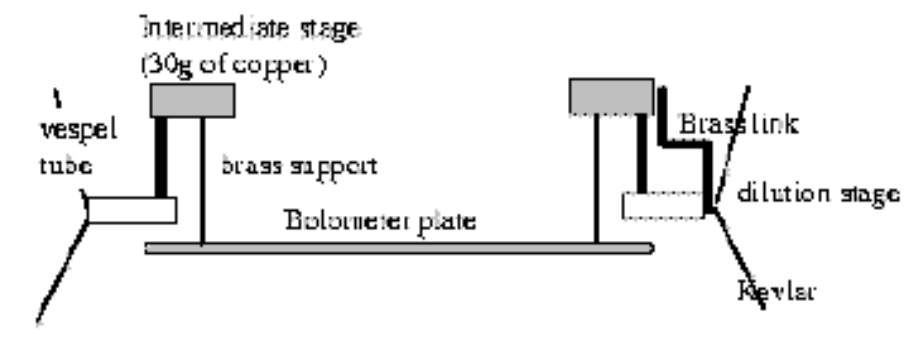

Fig. 12. Schematic drawing of the thermal filtering of the $100 \mathrm{mK}$ stage . 
tering is due to the bolometer plate specific heat itself $\left(\sim 10^{-3} \mathrm{~J} / \mathrm{K}\right)$. The time constant at this point is around 30 seconds. To obtain the best possible stability, we use a regulation on the intermediate stage (to suppress low frequency fluctuations) and passive filtering between the intermediate stage and the bolometer plate to suppress high frequency fluctuations. In fact, during the test flight, the dilution stage did not reach the base temperature, but stopped cooling at $112 \mathrm{mK}$ (because of a small leak in the cryostat). The regulation stage therefore remained inactive and we only used passive filtering.

\subsubsection{Thermometers}

Anderson insulators were used to measure the temperature at $100 \mathrm{mK}$. They were made at CSNSM Orsay [Dumoulin et al. , 1993]. These are thin layers of Niobium-Silicium deposited on saphire substrate and are very sensitive at $100 \mathrm{mK}$, where their impedance reaches a few $\mathrm{M} \Omega$. The read-out electronics are the same as for the bolometers.

\subsection{Stellar Sensor}

A custom star sensor has been developed for pointing reconstruction in order to be fast enough to work on a payload rotating at 2-3 rpm. At this spin rate, the use of a pointed platform for the star sensor is impractical. Each independent beam ( 8 arcmin wide) is scanned by the mm-wave telescope in about $10 \mathrm{~ms}$, establishing a detector response time that excludes the use of present large-format CCDs. We decided therefore to develop a simple night sensor, based on a telescope with photodiodes along the boresight of the $\mathrm{mm}-$ wave telescope. Thus, like the millimetre telescope, the star sensor scans the sky along a circle at an elevation of $41^{\circ} \pm 1^{\circ}$.

A linear array of 46 sensitive photodiodes (Hamamatsu S-4111-46Q) were placed in the focal plane of a $40 \mathrm{~cm}$ diameter, $1.8 \mathrm{~m}$ focal length parabolic optical mirror. Each photodiode has a sensitive area of $4 \mathrm{~mm}$ (in the scan direction) by $1 \mathrm{~mm}$ (pitch in the cross-scan direction). The dominant aberration of this simple telescope is coma, which is contained within a $0.2 \mathrm{~mm}$ diameter spot for the photodiodes at the edges of the array. The line of photodiodes is perpendicular to the scan (and so is aligned along elevation), and covers 1.4 degrees on the sky, with about 7.6 arcminutes (along the scan) by 1.9 arcminutes (cross-scan) per photodiode. A top baffle, painted black inside and located above all nearby payload structures, prevents stray radiation (direct and reflected from the gondola) from entering the telescope tube. A small baffle near the detector window further prevents stray radiation from reaching the photodiodes. 
During one rotation of the payload, the star sensor scans a full circle, $1.4^{\circ}$ wide at $41^{\circ}$ elevation. On average, this circle contains about 50 to 100 stars with magnitude brighter than 7 . The limiting magnitude of the sensor depends on the integration time available, as well as on the optical filters placed in front of the array (to reject parasitic signals) and on the electronic readout noise. For night-time operations of the test flight, we decided to avoid the use of optical filters in front of the sensor. The photodiodes are used in a photovoltaic configuration for minimal noise. The signal from the photodiodes is $\mathrm{AC}$ coupled (1.06 Hz cut-in frequency) to remove large-scale brightness gradients, and amplified by an low noise operational amplifier (OPA129P) connected as a current-to-voltage converter. The amplifier features a $1 \mathrm{G} \Omega$ feedback resistor with a $0.5 \mathrm{pF}$ capacitor in parallel to eliminate gain peaking. A second amplification stage $(\mathrm{G}=56)$ and a low-pass filter (second order, $33.1 \mathrm{~Hz}$ cut-off) are applyed to cover the dynamical range of a 12 bit A/D converter. The data are sampled 180 times per second and compressed (with loss) to 4 bits for each photodiode. In this configuration, the sensor can detect stars of magnitude 6-7 in $5 \mathrm{~ms}$ of integration.

The goal of the star detector software is to extract, from the time-sampled photodiode current values, candidate stars with detection time, measured flux, and quality criteria. This software should be able to run continuously during flight. For this we implemented an algorithm that treats independent photodiode data streams. The schematic of the algorithm is the following: for a given photodiode, when the program starts, it fills an array with the first 48 raw data samples and copies them to an "Analyzed Array" (AA). Among these, the first 32 samples form the "prepulse". The samples from 36 to 44 are named the "pulse array". Over the prepulse, we compute an average and a slope. We subtract these contributions from the Analysed Array, and then compute the root-mean-square noise over the prepulse (RMS). We search the pulse array for two consecutive samples larger than 6 times the RMS. If found, we look for the pulse maximum. We then compute the full-width at half-maximum, fit a parabola over the maximum and store the fitted time and amplitude. We may also compute, from the hardware description and rotation speed of the balloon, the expected pulse shape of an actual star event. We then fit this pulse shape to the data, and store a fitted amplitude, time and $\chi^{2}$ for the candidate star. We add this data to the list of time-ordered star candidates and make it available for the second step of the software - reconstruction of the telescope pointing. We iterate this algorithm over 46 photodiode data streams and then push the data streams by 8 samples forward. Selecting for clarity the largest amplitude star pulses, we plot in Fig. 13 the photodiode number of candidate star triggers versus time. Bright stars trigger the photodiodes at each rotation of the balloon over the sky. They drift over the photodiode array as the sky drifts in front of the balloon. From the trigger rate we deduce that the stellar sensor's limiting sensitivity is 6.6 magnitudes. 


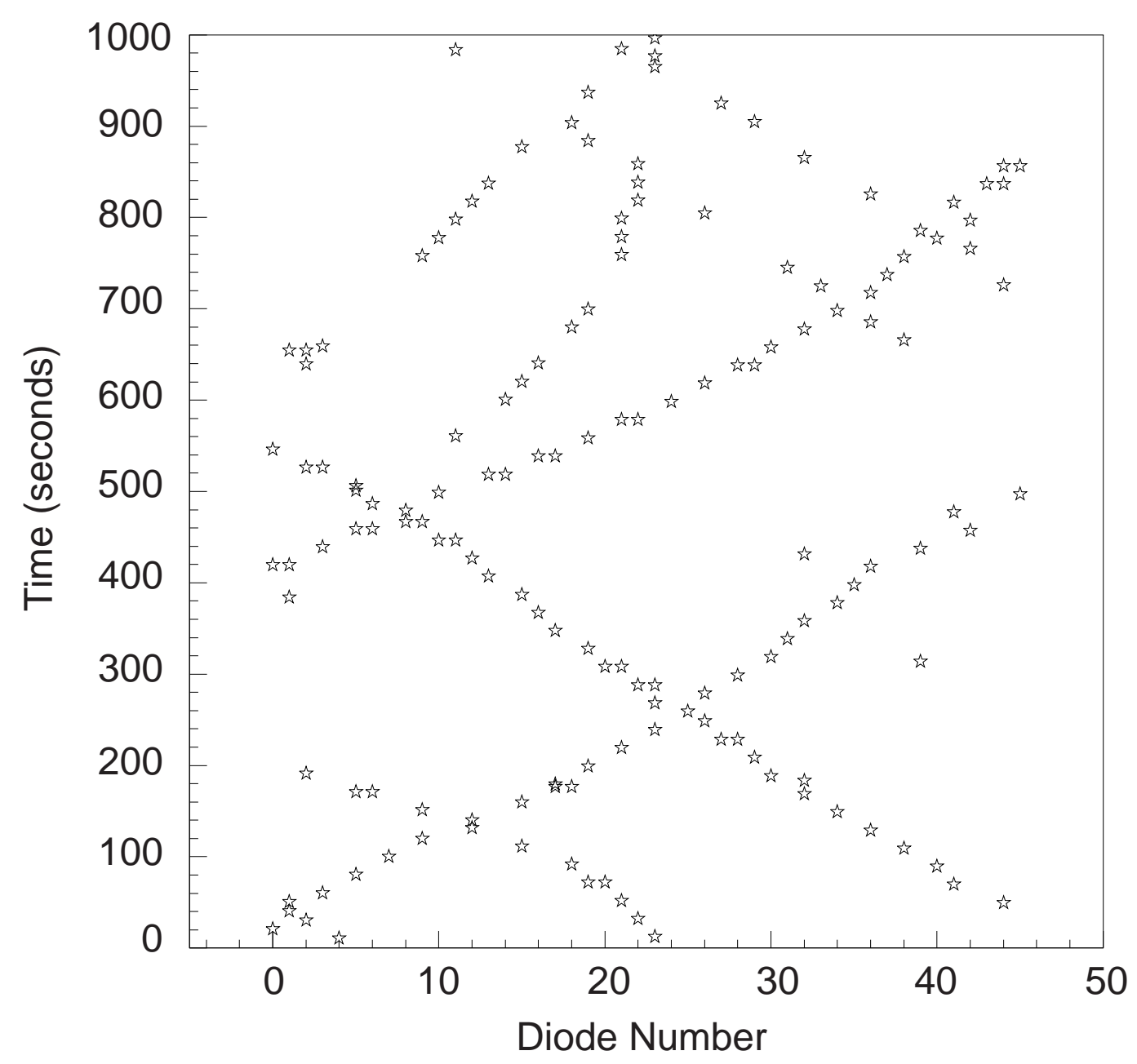

Fig. 13. This graph shows the diode number versus time of large amplitude candidates as seen by the star sensor. Bright stars trigger the star detector at every pass (20 second intervals). They are seen as "tracks" in this plot: as the sky moves in front of the balloon, star triggers move from one photodiode to the next.

\subsection{Gondola}

The gondola is composed of two frames (see Fig. 14). A stiff inner frame is needed to support the two mirrors and the photometer with negligible displacement under gravity, and subject only to self-similar scaling under temperature changes. It consists of welded 6061T aluminum $\mathrm{C}$ profiles (same material as the mirrors). The outer frame is lighter (built using aluminum square tubes $50 \times 50 \times 2 \mathrm{~mm}$ ) and connects the inner frame to the flight chain and supports the star sensor and navigation hardware (telemetry, ballast, batteries). The mass of the scientific part of the payload is $500 \mathrm{~kg}$; the total Trapani test flight payload mass at launch was $1000 \mathrm{~kg}$, including $250 \mathrm{~kg}$ of lead grain bal- 


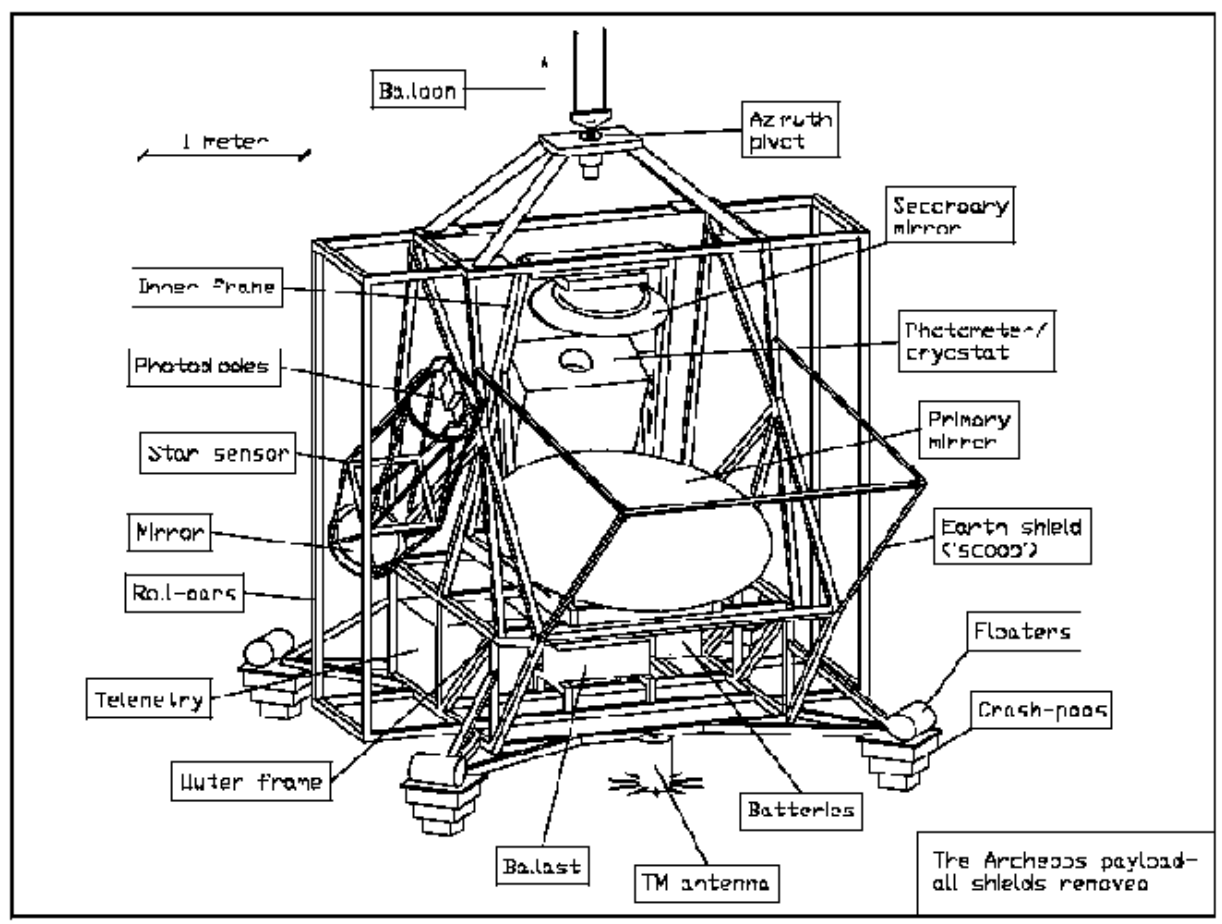

Fig. 14. Schematic functional drawing of the gondola

last. The inertial moment around the vertical axis is $I_{z z} \sim 1073 \mathrm{~kg} . \mathrm{m}^{2}$. The off-diagonal elements of the inertia matrix are $\sim 50$ times smaller than $I_{z z}$. $I_{x x}$ and $I_{y y}$ are 881 and $728 \mathrm{~kg} \cdot \mathrm{m}^{2}$, respectively. We thus expect pendulation resonances at $\sim 1 \mathrm{~Hz}$, significantly lower than the frequencies $(\gtrsim 4-6 \mathrm{~Hz})$ where the acoustic peaks of the CMB anisotropy are encoded by the 2-3 rpm azimuth scan.

The purpose of the Attitude Control System (ACS) is to spin in azimuth the entire payload at 2-3 rpm. To achieve this, we use an azimuth pivot mounted on top of the gondola. The pivot connects the flight chain of the balloon to the payload through a thrust bearing, providing the necessary degree of freedom for payload spin. Two deep groove bearings provide stiffness against transverse loads to the rotating steel shaft inside the pivot. A $60 \mathrm{~mm}$ diameter thrust bearing ensures little dynamical friction, of the order of $0.5 \mathrm{Nm}$ for a $2 \mathrm{rpm}$ rotation and 1 ton of suspended mass. The pivot includes a torque motor that acts against the flight chain to spin the payload. After initial acceleration, the motor provides just enough torque to compensate the friction in the thrust bearing and the small residual air friction. We use a motor with a torque constant of $0.24 \mathrm{Nm} / \mathrm{A}$. With a payload of 1 ton suspended below the pivot, this is enough to reach the nominal rotation speed in a few minutes, and to maintain it at $3 \mathrm{rpm}$ during the flight with an average current of $2 \mathrm{~A}$. The flight chain connecting the payload to the parachute and to the balloon is a 
long (> $10 \mathrm{~m}$ ) ladder made of two steel cables (separated by $0.5 \mathrm{~m}$ ) and rigid steps every 3 meters. When loaded with a 1 ton payload, this ladder acts as a spring with reaction constant $>50 \mathrm{Nm} / \mathrm{rad}$. This is stiff enough to apply all the needed torque without risks of twisting the ladder. The pivot is strong enough to withstand shocks of up to $10 \mathrm{~g}$ at parachute opening (with a 1 ton payload).

The rotation of the payload is monitored by a set of three orthogonal vibrating structure rate gyroscopes that can detect angular speeds as low as $0.1^{\circ} / \mathrm{s}$. These are sampled at $171 \mathrm{~Hz}$ by a 16 bit ADC. A PID feedback loop control is implemented in software, comparing the target azimuth velocity to the measured velocity. A phase modulated signal (PWM) to drive the pivot motor is synthesized from the error signal to drive the torque motor in the pivot. The PWM signal $(8 \mathrm{kHz})$ drives an $\mathrm{H}$ bridge of high power MOSfets. This powers the azimuth motor with high current (up to $7 \mathrm{~A}$ for tests and spin-up), while maintaining very low internal power dissipation.

\subsection{Electronics}

The following adjustable parameters are used to control the experimental setup; they correspond to commands that can be sent to the payload:

- modulation control for the 24 channels (bolometers and thermometers)

- focal plane temperature regulation heater

- rotation velocity for the gondola

- the main gate valve

- 6 heaters on the photometer box

The list of measurements:

- 24 channels for bolometer signals (sampled at $171 \mathrm{~Hz}$ )

- 8 channels for temperature measurements on the focal plane (sampled at $171 \mathrm{~Hz}$ )

- 4 channels for measuring the temperatures of the different screens (sampled at $171 \mathrm{~Hz}$ )

- 3 gyroscopes (sampled at $171 \mathrm{~Hz}$ )

- 46 stellar sensor diodes (sampled at $171 \mathrm{~Hz}$ )

- GPS data (time, position and altitude) (sampled at $0.5 \mathrm{~Hz}$ )

- 8 flow meters and pressure gauges for fluid control (sampled at $0.1 \mathrm{~Hz}$ )

- 6 thermometers inside the photometer box (sampled at $0.1 \mathrm{~Hz}$ )

- 8 thermometers for Helium level control (sampled at $0.1 \mathrm{~Hz}$ ) 


\subsubsection{Readout electronics}

The bolometers are biased using AC square waves by a capacitive current source. Their output is measured with a differential preamplifier and digitized before demodulation. We use the boxes already designed in preparation for Planck. Each box can manage 6 bolometers. During the Trapani test flight, we used 4 boxes for a total of 24 channels:

- 4 channels modified to measure the temperature of the different screens and to drive the heater currents.

- 20 channels using a cold JFET preamplifier:

- 9 channels for temperature measurements on the focal plane

- 6 bolometer channels

- 5 unused channels

All modulations are synchronous and driven by the same clock. This clock was also used for data readout, which was simultaneous for all bolometers and thermometers. Modulation parameters can be controled by telecommand. Sampling of the raw signal is at $6.51 \mathrm{kHz}$ before demodulation. Demodulation was performed by the EPLD and sampled twice per modulation period. We used a frequency of $85.7 \mathrm{~Hz}$ for modulation and $171.3 \mathrm{~Hz}$ for sampling.

\subsubsection{On-board computer}

The on-board computer consists of 3 cards:

- The main card (TCR) contains: the transputer T805 and an EPLD Altera 9400 to control communications and to directly drive the 6 readout boxes; the voltage regulators for the power supply; and the GPS module.

- the dilution card (DIL) contains: three rapid converters for the gyros, a phase modulated signal (PWM) to drive the pivot motor, one converter with a multiplexer for general control and a solid state relay to drive electrovalves and heaters. An EPLD Altera 9400 controls the card.

- the stellar sensor card (SST) contains: the 48 converters for stellar sensor data acquisition. An EPLD Altera 9400 controls the acquisition.

\subsubsection{On-board data storage}

After compression, the data are written to a storage module. The nominal option for this module is a 2 Gbyte Flash Eprom memory made of 256 circuits of 8 Mbytes each (we used only 1 GByte for the Trapani flight). A dedicated microprocessor is used to write the Eprom. The data storage module is installed in a sealed box, pressurized at 1 atmosphere. The data are read after retrieval of the balloon. 
Table 3

Data block description

\begin{tabular}{ccccl} 
block & $\begin{array}{c}\text { block size } \\
\text { type }\end{array}$ & $\begin{array}{c}\text { Trapani } \\
\text { bytes }\end{array}$ & $\begin{array}{c}\text { emitted blocks } \\
\%\end{array}$ & $\begin{array}{c}\text { flow rate } \\
\text { kbit/sec }\end{array}$ \\
\hline 1 & 136 & 10 & 0.258 & Onformation in block \\
2 & 276 & 7 & 0.366 & acq. status: clock, gain, modul.,... \\
3 & 260 & 2 & 0.098 & dilution status \\
4 & 96 & 10 & 0.182 & GPS character string \\
5 & 4240 & 2 & 1.6078 & 1-period samples: raw bolom. data \\
9 & 880 & 100 & 16.684 & gyroscopes \\
12 & 1384 & 100 & 26.240 & comp. bolom. (32 chan.*7 bit) \\
14 & 2404 & 100 & 8.6784 & comp. sensor data (4 bit/pixel) \\
\hline
\end{tabular}

\subsubsection{Batteries}

The power supply consists of 48 lithium batteries of $3 \mathrm{~V}$ and 36 Ah. This corresponds to a total power from 63 to $100 \mathrm{~W}$. The theoretical duration of the batteries is longer than 45 hours for each part. The batteries are mounted together in an insulated box with a heater to maintain the temperature above $-10^{\circ}$ C. A switching system allows us to save the system in the event of a battery failure.

\subsection{Data Aquisition}

\subsubsection{Data flow between the experiment and the transputer (link1)}

The sampling frequency may be adjusted from $100 \mathrm{~Hz}$ to $200 \mathrm{~Hz}$ and corresponds to a period $P_{\mathrm{s}}$ of 5 to $10 \mathrm{~ms}$ (corresponding to half a modulation period). The number of digitisations, $N_{\mathrm{d}}$, over half the modulation period can be adjusted from 36 to 48, yielding a digitisation period $P_{\mathrm{d}}$ of between 100 and $280 \mu \mathrm{s}$. At this rate the EPLD sends data to the transputer as 64 bit words, corresponding to a data flow of 230 to $640 \mathrm{kbit} / \mathrm{sec}$.

Only the raw data of a single bolometer is transmitted at a time; after each full modulation period $\left(P_{\mathrm{m}}=2 P_{\mathrm{s}}\right)$, the acquisition process moves on to the next bolometer. This corresponds to a cycle of 36 periods and results in 2.5 measurements/sec:

- The stellar sensor data: 
48 ADC values of 12 bits are read at a time, giving 576 bits. They are sent during 36 digitisations with 16 bit words.

- The bolometer measurement after demodulation: the sum of the bolometer values (16 bits) during one half period $(\max 48=6$ bits) gives a 22 bit number. The LSB bit is lost and 36 words of 21 bits, corresponding to the 36 measuring channels, are successively sent during one sampling period (half a modulation period);

- The synchronisation bits: Two synchronization bits allow data verification and permit the identification of any problems in the acquisition chain. The transputer uses these bits to re-synchronise the data;

- The status of valves and heaters: One bit per word gives a sequence of 72 bits in one period. This sequence encodes the status of all switches (heaters, valves);

- The gyros, GPS, temperatures, pressures and flow: during one half period, this byte contains:

- the even bytes contain one GPS character, if any.

- bytes 1 and 3: the $\mathrm{X}$ gyroscope data

- bytes 5 and 7: the Y gyroscope data

- bytes 9 and 11: the Z gyroscope data

- bytes 13 and 15: the multiplexed data from the dilution board

- byte 17: address of the multiplexed data.

The multiplexed data switch between 48 different measurements.

\subsubsection{Data formatting and compression in the transputer}

The data are transformed by the transputer into blocks of different types, each block structure containing some partial information. A separate task is the transformation of a block into a smaller one containing the same information after compression. Tab. 3 describes the different block types and gives their size, percentage of the block emitted, flow rate in kbit/second and the information content. These parameters change during experimental testing and can be modified for flight; the table shows values appropriate for the Trapani test flight, where the total flow rate was $84 \mathrm{kbit} /$ second, sent as a bi-phase signal at $108 \mathrm{kbit} /$ second via telemetry for real-time control. The same data are stored in the flash EPROM on-board recorder, where a volume of 1 Gbit can be stored during 24 hours of acquisition. For the Kiruna flight, the typical flow rate will correspond to 2.5 blocks per second (modulation $90 \mathrm{~Hz}$ ). The total storage required is calculated for 30 hours of observation. The total compressed data include blocks $12-14$ and blocks $1-2-3-4-5$ every 4 seconds (1/10 of the blocks). 


\subsubsection{Data flow between the transputer and the telemetry (link2)}

For ground-based testing and calibration, telemetry is passed through an optical fiber at a rate of $1 \mathrm{Mbit} / \mathrm{s}$. Thus, it is possible to transmit all information without compression from the experiment.

\section{Ground-based calibration}

Ground tests were planned before the Trapani test flight to photometrically and optically calibrate the instrument. Unfortunately, due to the late integration (May 1999) just before the test flight (July 1999), only methodology could be validated, without any complete end-to-end results ${ }^{4}$. Nevertheless, performance was judged to be sufficient for the test flight. Here we describe the present status of the ground-based calibration devices.

\subsection{Instrument photometric performance}

In order to calibrate total transmission, it was decided to use a cold blackbody (CBB) with a variable temperature in front of the entrance window of the photometer (with only the thin polypropylene membrane as a separator). We deduce the total transmission of the photometer by varying the temperature of the CBB, filling the beams, and measuring the I-V curves. Here we describe the calibration cryostat that was designed for this purpose. A schematic drawing is shown in Fig. 15. The cryostat can be attached even when the photometer is on the gondola (at an inclination of 28 degrees) to permit calibration just before flight. The cryostat is cooled with liquid Helium only. Helium vapours cool an intermediate screen (IS) and then go through a heating plate in order to avoid air freezing at the exit, thereby preventing water from dripping onto the primary mirror. The CBB plate itself is made of copper covered with a black material [Lee et al., 2000]. It is coupled via three copper bands to the liquid He tank. The blackbody temperature is changed (over a range from 6 and $25 \mathrm{~K}$ ) via three resistances (with up to 5 Watts of input power). To match the various experimental conditions (optical background load due to various emissivities), the thermal copper links must be appropriately adapted. Heating the CBB causes more He evaporation, which then reduces the IS temperature and modifies the optical background on the $10 \mathrm{~K}$ photometer stage.

This calibration cryostat has a built-in light pipe that can feed a modulated external source through small holes in the $\mathrm{CBB}$, in order to measure the time

$\overline{4}$ The optical ground-based tests have been obtained since (July 2000) 


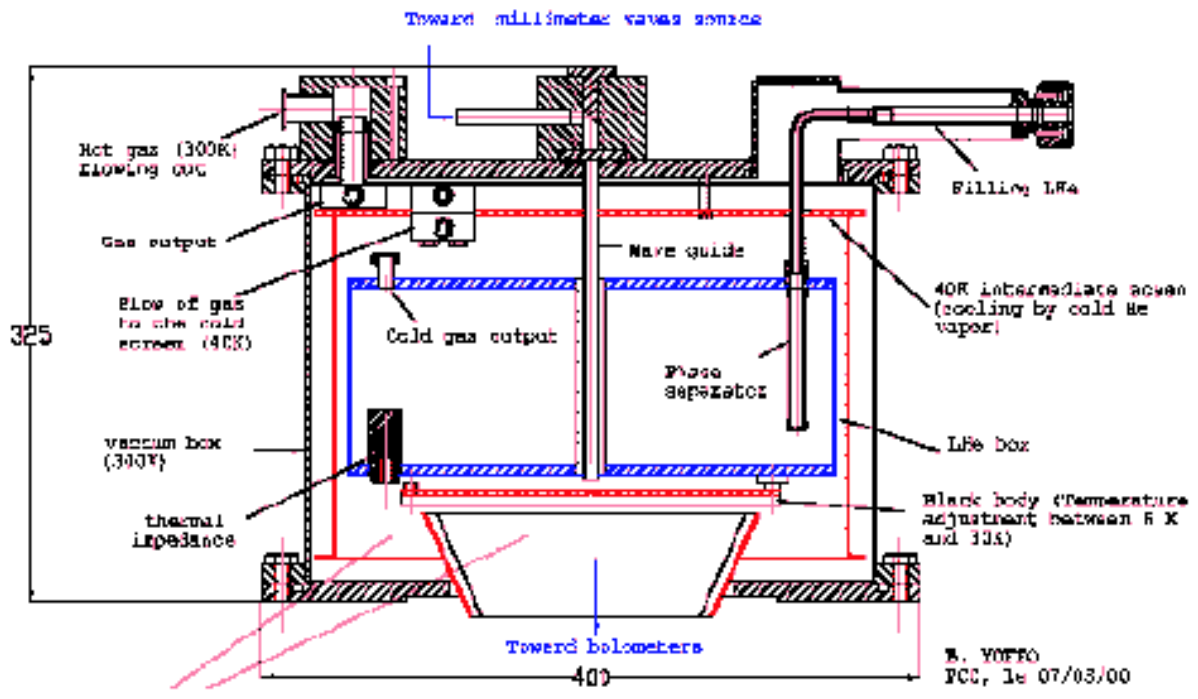

Fig. 15. Schematic drawing of the calibration cryostat. This is bolted to the photometer during the ground-based calibration on top of the gate valve (Fig. 11. Dimensions are in millimetre. 
constants of the detectors (see Fig. 15). This measurement is important for this kind of scan-modulated CMB experiment, in order to assess the linearity of the bolometer response as a function of frequency.

The photometer for the Trapani 1999 campaign demonstrated satisfactory behaviour under the background conditions provided by the calibration cryostat (at typically $10 \mathrm{~K}$ ), which were similar to what was expected for the flight.

No Fourier Transform Spectrometer (FTS) measurements have yet been performed to check the photometric pixels spectral transmission. For the interpretation of the test flight data, we have to rely on the composite transmission as deduced from the single filter and back-to-back horn transmissions (see Sec. 2.2 and Fig. 8).

\subsection{Instrument optical calibration}

A pointing table has been built at the ISN laboratory in order to perform a ground-based determination of the main lobe of the optical system. The gondola is mounted on this platform. A software has been developped to pilot the pointing device in order to scan an area containing a thermal source (placed on a hill at typically $1 \mathrm{~km}$ distance).

For this purpose an extended modulated source $\left(\simeq 1 \mathrm{~m}^{2}\right)$ has been constructed of which an exploded scheme is shown on Fig. 16.

This source has a regulated differential temperature of $\Delta T=50 \mathrm{~K}$. The modulation is triangular. With a typical rotation period of $0.5 \mathrm{~s}$, the modulated signal has a frequency of $8 \mathrm{~Hz}$ (the source has 4 quadrants: see Fig. 16), which is easily detected by simple lockin on the bolometer signal with respect to the thermal background. The source has been tested at the Plateau de Bure observatory by measuring the main lobe of the POM2 telescope coupled to the Diabolo cryostat [Benoît et al., 2000]. Some preliminary measurements were performed at close positions at Trapani just before the flight.

The very tight schedule before the Trapani flight did not allow optimization of all the elements specially conceived for the ground-based calibration: pointing table, software, extended source, for the technical flight. Eventually, the optical calibration system was successfully tested end-to-end in July 2000 and produced beam sizes of about 8 arcminutes at all frequency bands. This sytem will be used for the Kiruna flights. In flight measurements of the beam sizes during the Trapani campaign are discussed in section 5.2.3. 


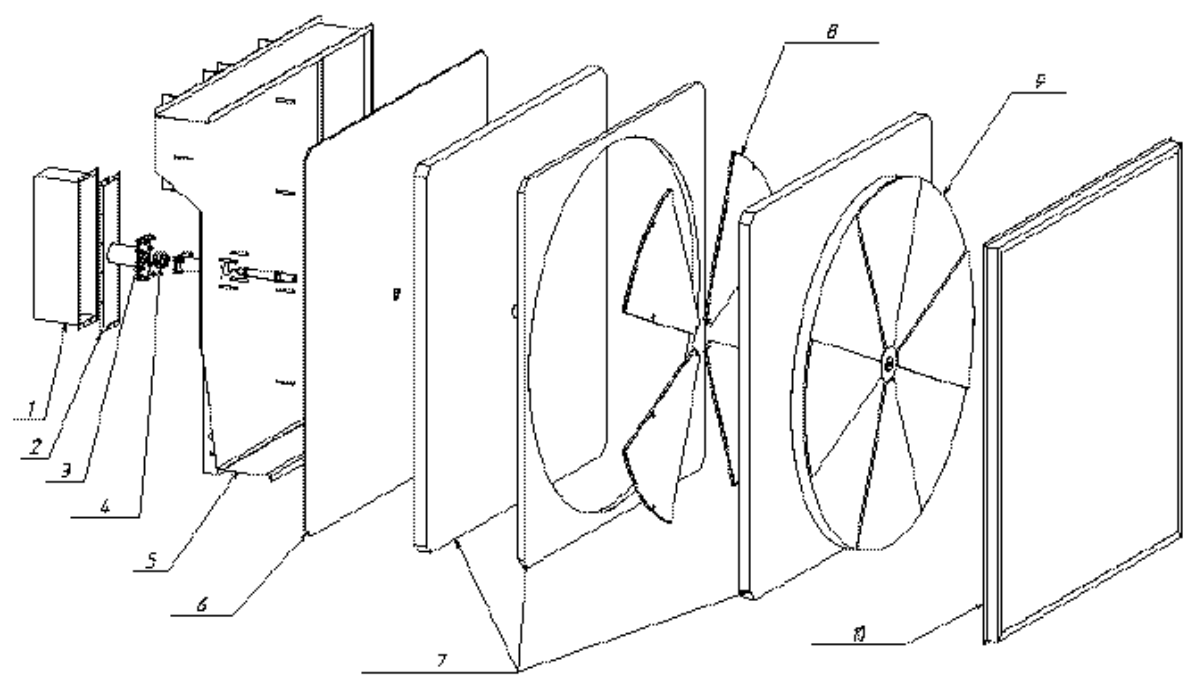

Fig. 16. The modulated source in exploded view. 1: cover, 2: gasket, 3: connections, 4: engine, 5: box, 6: Eccosorb background, 7: heat insulators (polystyrene), 8: heating sectors \& Eccosorb, 9: chopper (polystyrene wheel + Eccosorb), 10: cover plate. A detector placed far above (in this figure) will observe alternating hot (8) and cold (6) loads as the chopper (9) is rotated.

\section{Trapani Test Flight: Description}

\subsection{General timing}

Launch of the balloon was provided by ASI (Italian Space Agency) base near Trapani (Sicily) in the evening of the 17th July 1999 at the end of the scheduled launch window, in particular when the meteorological requirements were met. A logbook summary is given here with hours given in universal time (decimal hours starting on the 17th) and local time (hours and minutes) as shown in parenthesis:

$19.37(21: 22)$ : launch

21.92 (23:55) : PID adjustement of the pivot; the gondola starts to spin at $3 \mathrm{rpm}$ (13.6 deg/sec for a beam at 41 degree elevation)

$21.97(23: 58)$ : opening of the protection valve in front of the membrane (Fig. 11) and the cold optics (this valve opens automatically when the pressure outside the cryostat drops below $20 \mathrm{mb}$ )

23.00 (01:00) : Galaxy detected by all bolometers 
$23.45(01: 27)$ : standard altitude achieved; cryostat temperature stabilizes at $112 \mathrm{mK}$ : final polarisation adjustment for the bolometers

28.00 (06:00) : Sunrise

$31.53(09: 32)$ : protection valve closes automatically when the temperature of the $10 \mathrm{~K}$ stage reaches $13 \mathrm{~K}$ due to Sun heating.

34.00 (12:00) : ${ }^{3} \mathrm{He}$ bottle empty

$36.32(14: 22)$ : separation of the gondola from the balloon.

$37.36(15: 23)$ : landing in Spain

40.22 (18:13) : liquid Helium cryostat empty

\subsection{Flight Characteristics}

The balloon trajectory was nominal for this trans-Mediterranean flight. After take-off from Trapani (Sicily) the balloon grazed the African continent before reaching Spain. Altitude was between 40 and $42 \mathrm{~km}$ during the useful flight (UT $=24$ to 36 hours)

Due probably to a small leak in the cryostat, the minimum temperature reached was only $112 \mathrm{mK}$ and could be obtained only with a higher than normal flow rate of $24 \mu \mathrm{mol} / \mathrm{s}$ for ${ }^{4} \mathrm{He}$ instead of $16 \mu \mathrm{mol} / \mathrm{s}$, and $8 \mu \mathrm{mol} / \mathrm{s}$ instead of $4 \mu \mathrm{mol} / \mathrm{s}$ for ${ }^{3} \mathrm{He}$. The temperatures of the different stages are represented in Fig. 17: during the scientific observing time (from 24 to $28 \mathrm{hr}$.), all temperatures are relatively stable. The $1.6 \mathrm{~K}$ stage fluctuates with an amplitude of $\sim 10 \mathrm{mK}$ at frequencies around $2.5 \times 10^{-3} \mathrm{~Hz}$; the long-term temperature increase was about $60 \mathrm{mK}$ over the entire scientific observing time. The temperature of the $10 \mathrm{~K}$ stage decreased from 10 to $9 \mathrm{~K}$ during this period, followed by a large increase after Sunrise. The $100 \mathrm{mK}$ stage shows a fluctuation of $1.9 \mathrm{mK}$ (peak to peak). Temperatures for the different filtering stages at $100 \mathrm{mK}$ (Fig. 12) are given in Fig. 18, and the fluctuation spectra of these stages are shown on Fig. 19. Thermal noise at bolometer level resides now only at low frequency, while the high frequency white component is due to thermometer noise.

\section{Trapani Test Flight: in-flight performance}

\subsection{Bolometer signals}

One of the $143 \mathrm{GHz}$ photometric pixels (B1-2) failed early during the night and another at $217 \mathrm{GHz}$ (B2-5) was very noisy. Both problems were due to faulty connections in the electronic system. The other four bolometers delivered a 

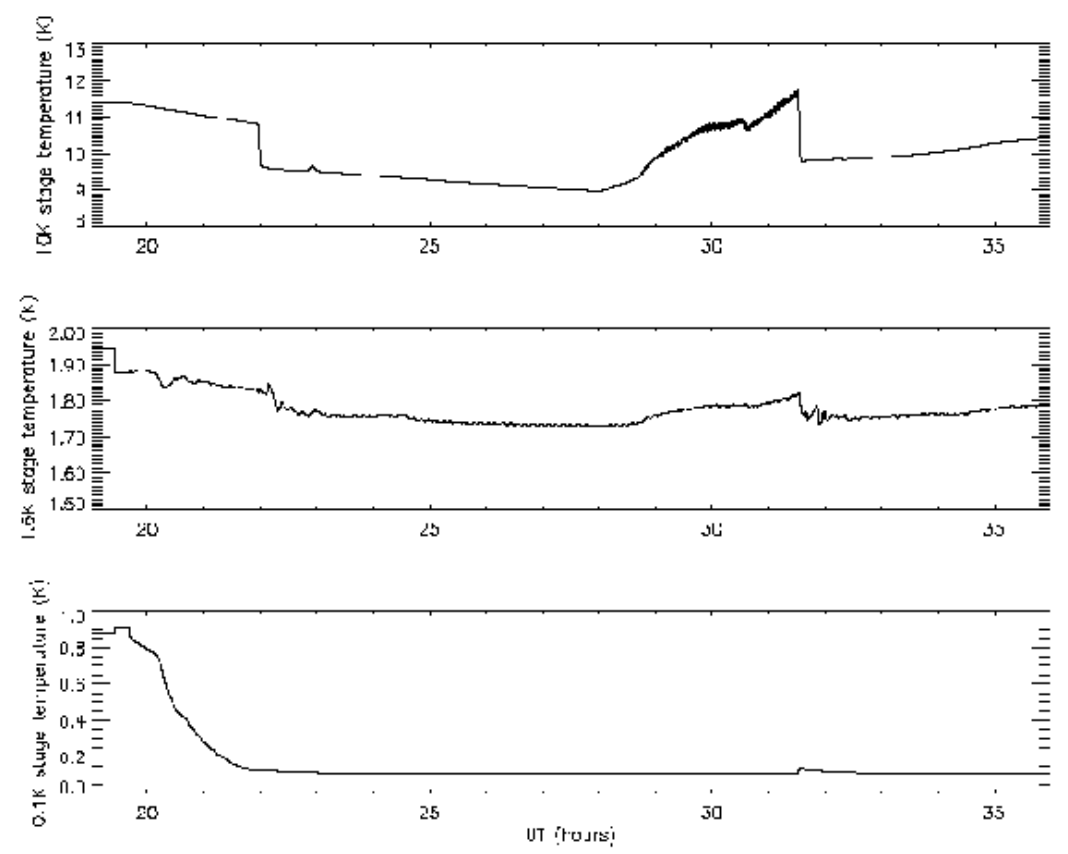

Fig. 17. Measured temperature of the different stages as a function of universal time during the flight. The drop of temperature of the $10 \mathrm{~K}$ stage happens when the valve opens. After sunrise at UT $=28 \mathrm{hr}$, this stage starts to warm up until the valve is shut after $\mathrm{UT}=31.5 \mathrm{hr}$.

continuous and satisfactory signal during the whole flight. Fig. 20 shows the filtered signal from 3 of them for few minutes of data. The sharp peaks in the valleys of each oscillations come from the emission of our galaxy. The CMB dipole is present at about half the level of the oscillations in the $143 \mathrm{GHz}$ channels (see subsection 6.3).

The main oscillations in the signal in Fig. 20 are due to a persistent parasitic signal which was present in all bands (with a high correlation between the bands) during the Trapani flight. It appears as a strong sine-like oscillation at the spin frequency in the data and systematically shows two smaller bumps about 70 degrees apart from the maximum signal. The phase of this signal is not constant relative to fixed geographical directions or celestial coordinates but slowly drifts in a non monotonous fashion by about 1.5 turn during the four hours of night data. The origin of this signal is not fully understood. It is not in phase with variations of the elevation of the sub-millimeter axis as deduced from preliminary reconstruction using the stellar sensor information. We therefore can exclude that this parasitic signal is induced by the swing motion of the gondola. Intrinsic changes in the atmospheric emission with direction, such as the presence of clouds with enhanced ozone abundance, can also be excluded since they would require rather high abundance changes and 


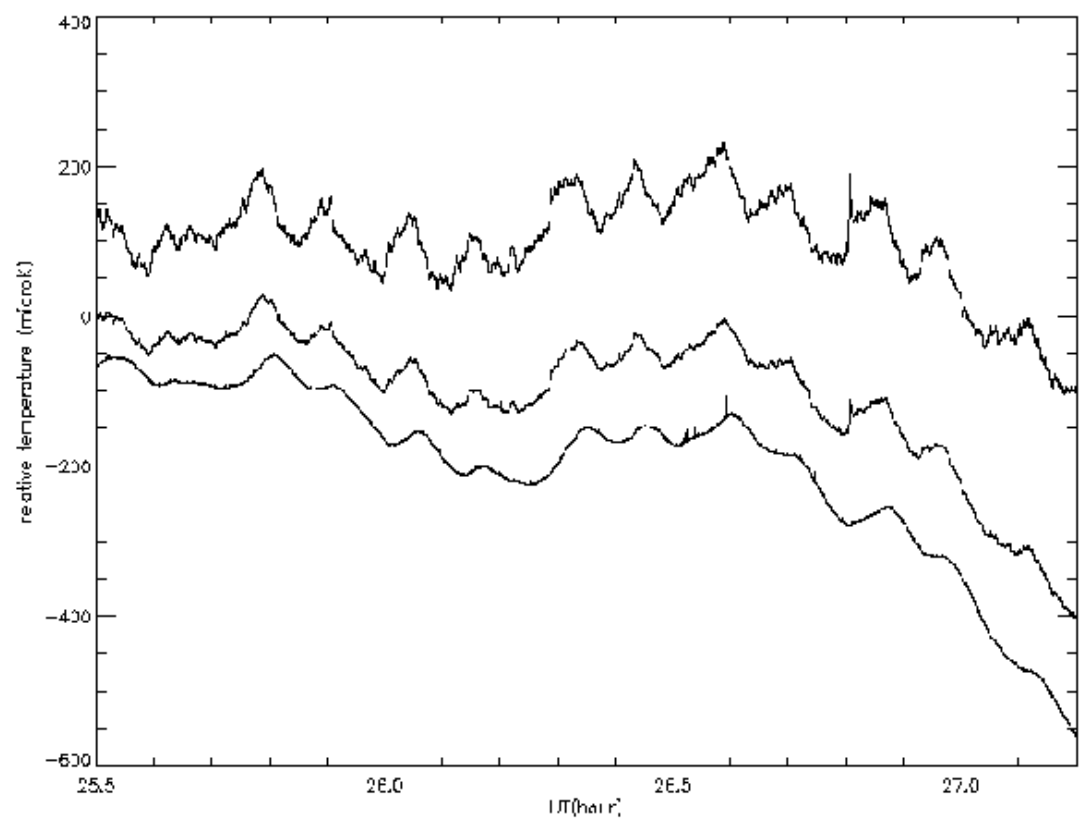

Fig. 18. Relative temperature fluctuation of the dilution part of the cryostat as a fuction of universal time. The top curve is for the dilution stage, the middle for the intermediate stage and the bottom curve for the bolometer plate (see Fig. 12).

a rather unrealistic cloud distribution in order to reproduce the phase drift observed. A more likely hypothesis is that this signal is actually due to nonsymmetrical emission or reflection of the atmospheric or earth emission by the balloon, seen through the far-side lobes of the instrument. This has lead to a new design of the entrance baffle of the experiment for the forthcoming polar flight from Kiruna. The charaterisation and removal of this parasitic signal is one of the main data reduction task ahead.

\subsection{Pointing reconstruction}

\subsubsection{Pointing solution from Galaxy crossings}

As a first step in the analysis, we obtained an approximate pointing solution for the experiment using the bright Galactic plane crossing at each rotation of the gondola. We assumed that there was no pendulation of the gondola. The IRAS maps were used to compute the azimuth of the Galactic plane each time it was crossed by the Archeops $353 \mathrm{GHz}$ beam, accounting for the location of the gondola (given by the GPS). We could therefore synchronize the azimuth once per rotation during the flight. We then interpolated between 


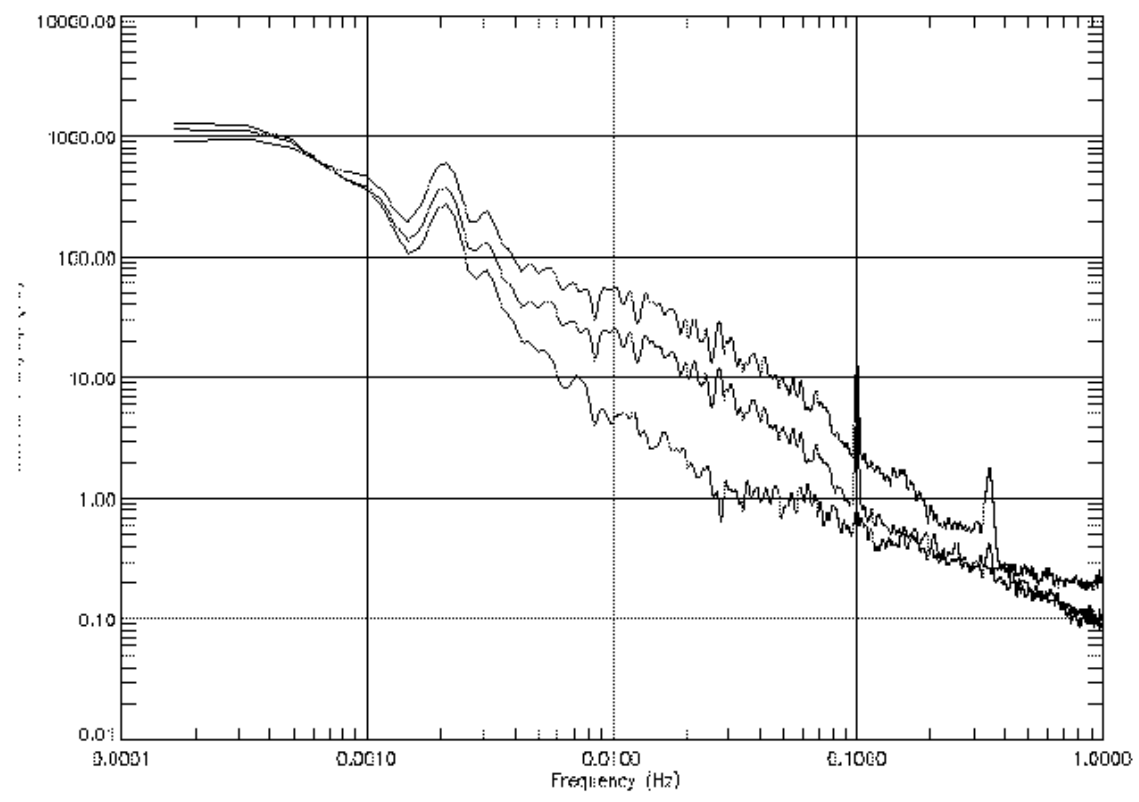

Fig. 19. Power spectrum of thermal fluctuations at $100 \mathrm{mK}$. The different curves represent the fluctuations on the dilution stage, on the intermediate stage and on the bolometer plate.

these Galaxy crossings in order to obtain an approximate pointing solution. We estimate the accuracy of this pointing solution to be about 30 arcminutes.

\subsubsection{Pointing reconstruction from the Fast Stellar Sensor}

The final reconstruction of the telescope attitude will be obtained in three steps: 1) finding star candidates in the data stream, 2) associate these to wellknown stars and 3) relate the solution to the submillimetre beams. The first step is described in Sect. 2.4. The second step has yielded preliminary results that are used in the following. Final refinements are now being done and will be reported soon. The third step is described now.

\subsubsection{Focal plane geometry and beams}

We made scans of Jupiter when it crossed an elevation of 41 degrees at $2 \mathrm{~h} 48$ UT on July 18th, 1999, when the balloon was approximately above Algiers. Jupiter's azimuth was 110 degrees. Due to our scan strategy, Jupiter appears as a peak in the bolometer signal at each rotation when it passes through the focal plane. Jupiter has an angular diameter of less than 40 arcseconds and is therefore much smaller than our beams. We may therefore consider it as 

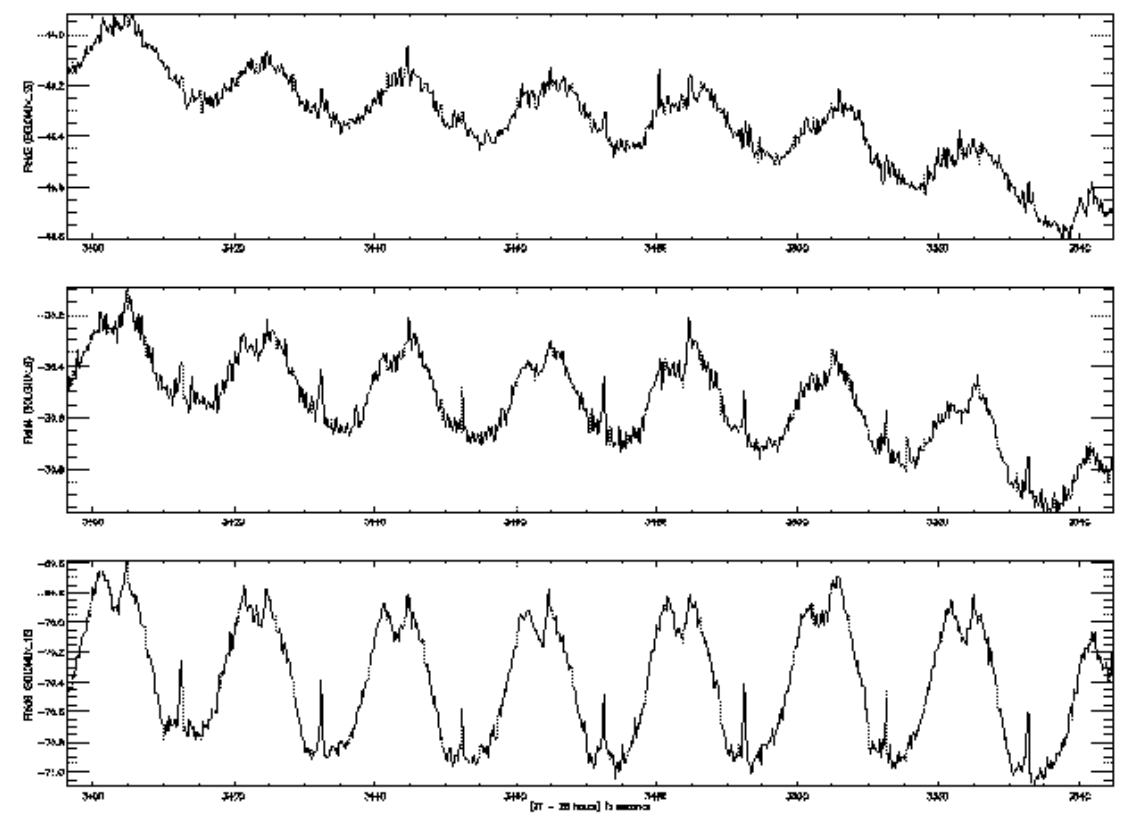

Fig. 20. Signal in $\mu$ Volts from three bolometers as a function of time (seconds). From top to bottom, the three channels are shown $(143,217$, and $353 \mathrm{GHz}$ ) for about 8 revolutions of the gondola. The signal was deglitched and low-pass filtered $\left(f_{c} \simeq 3 \mathrm{~Hz}\right)$ to show its broad features.

a point source for the purpose of mapping the beams and reconstructing the focal plane geometry.

We extract the focal plane geometry by measuring the relative shift in azimuth and elevation of each bolometer between the observed signal and the true position of Jupiter on the sky. The fast star sensor analysis provided sky coordinates for a point located roughly in the middle of the focal plane with an accuracy of about 3 arcminutes. The focal plane geometry is then measured relative to that point for each bolometer by fitting the location of a 2-dimensional Gaussian to the image of Jupiter. In-scan FWHM of $11 \operatorname{arcminutes}( \pm 1)$ are found for all bolometers while cross-scan FWHM of 13 arcminutes are measured except for bolometer 3-6 (24 arcminutes reflect the double-peaked structure).

The beam maps were obtained by projecting the data from each bolometer using a preliminary attitude reconstruction from the fast stellar sensor around the location of Jupiter. Fig. 21 shows the measured beams for our bolometers and their relative positions in the focal plane. We emphasize that our scan strategy provides only a few passes across Jupiter for every photometric pixel, which typically give less than 10 detector samples within a two dimensional 


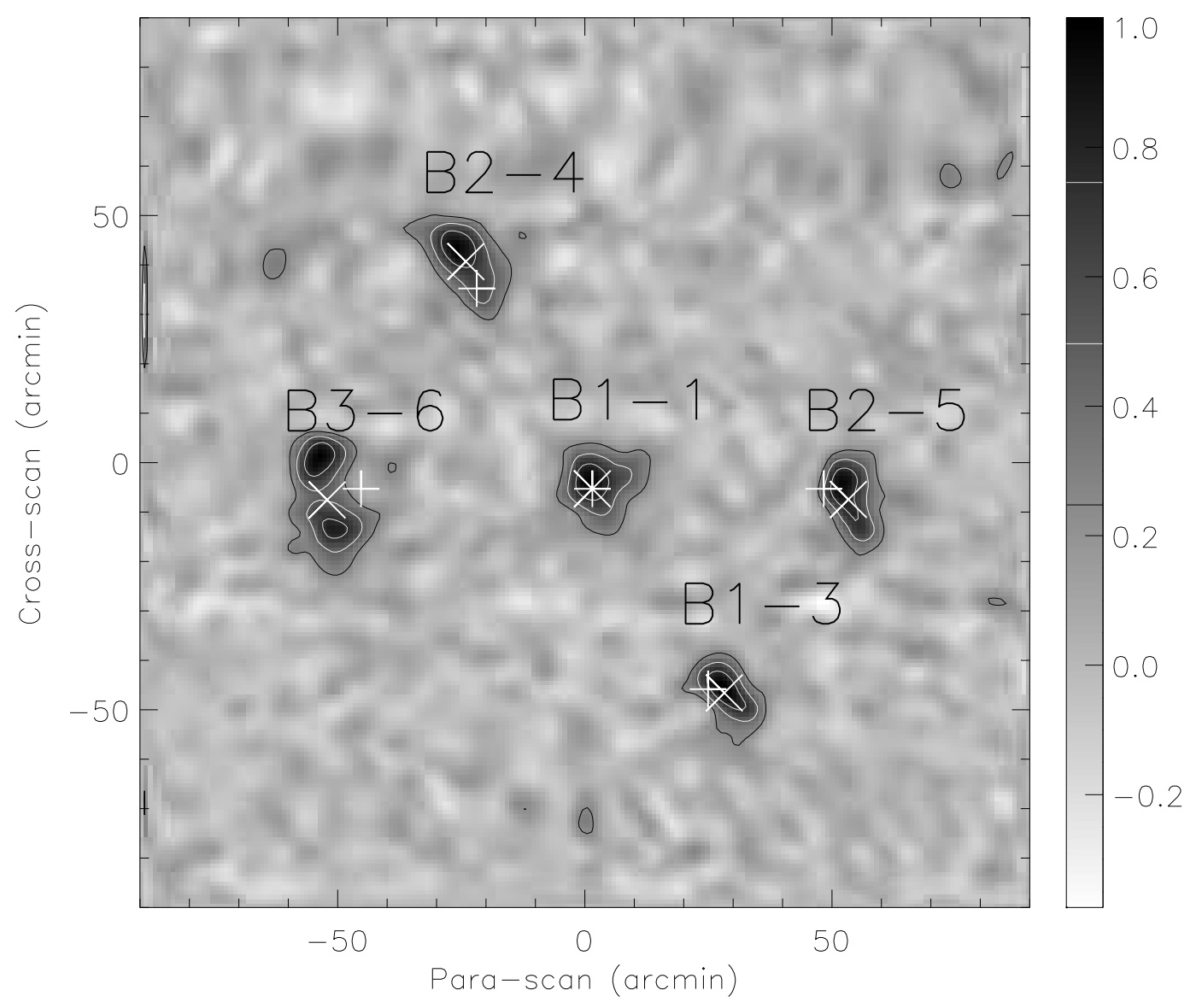

Fig. 21. The focal plane configuration during the Archeops flight in Trapani and the measured beam shapes. The white pluses show the expected location of the photometers relative to the center one. The white crosses are the centers of a Gaussian fit to the measured beams (preliminary results). Photometer B1-2 did not work during the flight and is therefore not shown in the figure.

full-width at half-maximum. Therefore, the beam maps are noisy and are sample limited, and the reconstructed sizes and shapes should be considered with caution. The double peaked shape of bolometer 3-6 seems, however, to be real as it is also observed in the Saturn scans. A misalignment in the optical components of the telescope or focal plane optics could produce the double peaked shape, as well as asymmetry in the beams.

\section{In-flight photometric calibration}

We devised 3 schemes to independently calibrate the photometry of the experiment. The final CMB calibration comes from the CMB dipole because it has the same spectrum as the CMB anisotropies. Other methods allow to check the consistency of the calibration accross the $l$ spectrum and allow the calibration of "non-CMB" channels. 
Table 4

Preliminary calibration values in $\mathrm{mK}_{R J} / \mu \mathrm{V}$ (error bars will be refined but are below $\left.2 \mathrm{mK}_{R J} / \mu \mathrm{V}\right)$

\begin{tabular}{|l|l|l|l|l|}
\hline bolometer & $1-1$ & $1-3$ & $2-4$ & $3-6$ \\
frequency $(\mathrm{GHz})$ & 143 & 143 & 217 & 353 \\
\hline Galaxy & 12.2 & 11.1 & 6.3 & 6.4 \\
Jupiter & 15.3 & 15.3 & 8.7 & 8.1 \\
Dipole & 12.2 & 14.7 & 6.7 & - \\
\hline
\end{tabular}

\subsection{Photometric calibration on Jupiter}

As Jupiter is much smaller than our beam it can be used as a point source for calibration. We assume a temperature of $T_{\mathrm{RJ}}^{p}=170 \mathrm{~K}_{\mathrm{RJ}}$ [Goldin et al., 1996]. The calibration coefficient is obtained through:

$$
\alpha=\frac{T_{\mathrm{RJ}}^{p} \times \Omega_{p}}{I_{p}} \quad\left[\mathrm{mK}_{\mathrm{RJ}} \cdot \mu V^{-1}\right]
$$

where $I_{p}$ is the total measured integrated flux of Jupiter in $\mu \mathrm{V}$. $\operatorname{arcmin}^{2}$ and $\Omega_{p}$ is the known solid angle (in $\operatorname{arcmin}^{2}$ ) subtended by the planet. The results obtained with Jupiter for the calibration are shown in Tab. 4.

\subsection{Photometric calibration on the Galaxy}

In-flight calibration of the three Archeops bands was done on the Galaxy using FIRAS data. We first constructed maps at 143, 217 and $353 \mathrm{GHz}$ by fitting FIRAS spectra with Planck curves modified by $\nu^{\alpha}$ emissivity laws. Archeops Galactic maps where then convolved with the FIRAS point spread function and color corrected using the FIRAS-fitted parameters. For selected longitudes with good signal-to-noise ratios, we computed the Archeops and FIRAS Galactic profiles in latitude $\left(-30^{\circ}<b<30^{\circ}\right)$. These profiles were fitted by Gaussian curves, and calibration coefficients obtained by comparing the heights of these two curves.

At each selected longitude, the shapes of the two profiles show good agreement (see an example in Fig. 22). There is a small dispersion in calibration coefficients from point to point. Derived values are in Tab. 4. 


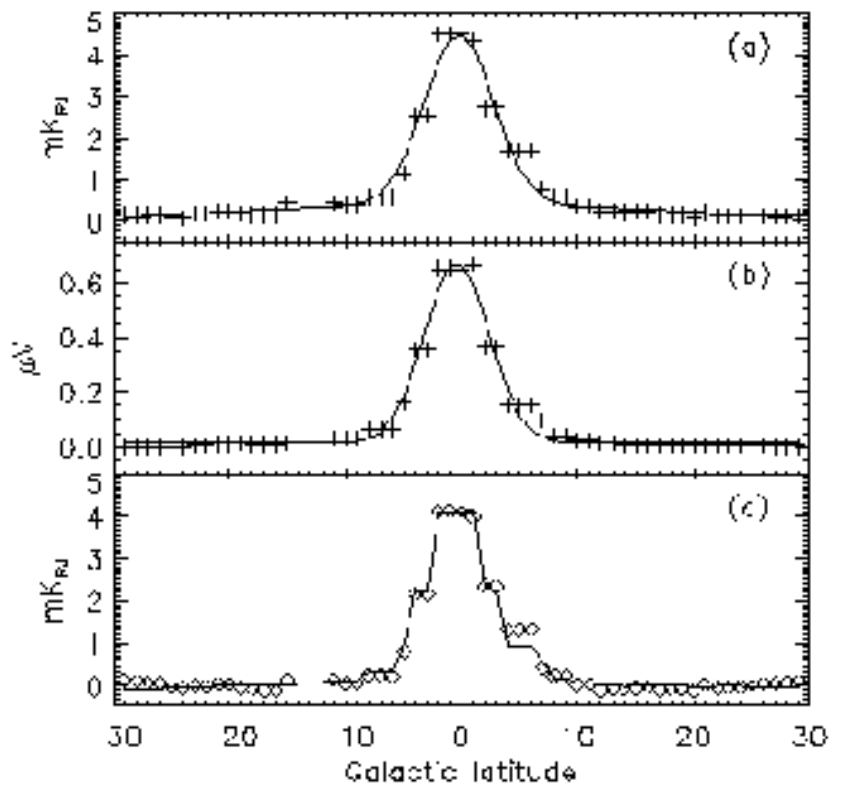

Fig. 22. Exemple of cross-calibration between Archeops and FIRAS data at $353 \mathrm{GHz}$ (at Galactic longitude $\mathrm{l}=33^{\circ}$ ). (a): FIRAS Galactic latitude profile (crosses) and its Gaussian fit (continuous line) (b): ARCHEOPS Galactic latitude profile (crosses) and its Gaussian fit (continuous line) (c): FIRAS Galactic latitude profile (diamonds) together with the Archeops one (continuous line) multiplied by the calibration factor of $6.4 \mathrm{mK}_{R J} / \mu \mathrm{V}$. 


\subsection{Photometric calibration on the CMB Dipole}

Using the 850 micron band $(353 \mathrm{GHz})$ as a template for the parasitic signal, and assuming that the lower frequency channel contains only a linear combination of the parasitic and dipole signal (as known from COBE data) one obtains the dipole calibration shown in Tab. 4. We note that the dipole calibration accuracy is well below what can be expected ultimately, probably because it is still hampered by unidentified signal near the spin frequency in the data, and is by no means final.

\subsection{Noise}

The final sensitivity of the bolometers during the test flight can be estimated as (for the white noise at high frequency) $130,67,64 \mu \mathrm{K}_{R J} \mathrm{~Hz}^{-1 / 2}$ equivalent to 220, 190, $740 \mu \mathrm{K}_{C M B} \mathrm{~Hz}^{-1 / 2}$ at 143,217 , and $353 \mathrm{GHz}(2.1,1.4$, and $0.85 \mathrm{~mm}$ ) respectively. With 3 bolometers on the $\mathrm{CMB}$ sensitive frequencies one can expect an instrument $\mathrm{CMB}$ sensitivity of $120 \mu \mathrm{K}_{C M B} \mathrm{~Hz}^{-1 / 2}$ during the 4 hours in dark night. These sensitivities are very similar to the ones obtained in other balloon-borne experiments like Boomerang [de Bernardis et al., 2000] and Maxima [Hanany et al., 2000]. The gain brought by the $100 \mathrm{mK}$ stage is mainly to allow fast bolometers (at most few milliseconds of time constant). Understanding the full power spectrum of the bolometer noise is one of the main tasks of the data reduction.

\subsection{Sky coverage}

During the 4 hours of dark night for the Trapani flight (valid for CMB analysis), the observed sky is as shown in Fig. 23. It covers about $19 \%$ of the total sky.

\section{Conclusion}

We have shown the main technical description of the Archeops balloon experiment. Then we present the status and preliminary results from the test flight in Trapani July 1999. It is the first time a dilution fridge has been embarked on a balloon flight. This is an important result because the dilution cryostat is very similar to the one in Planck HFI. It is also the first time that 3 independent calibration methods are used (planet, dipole and Galaxy). They give 


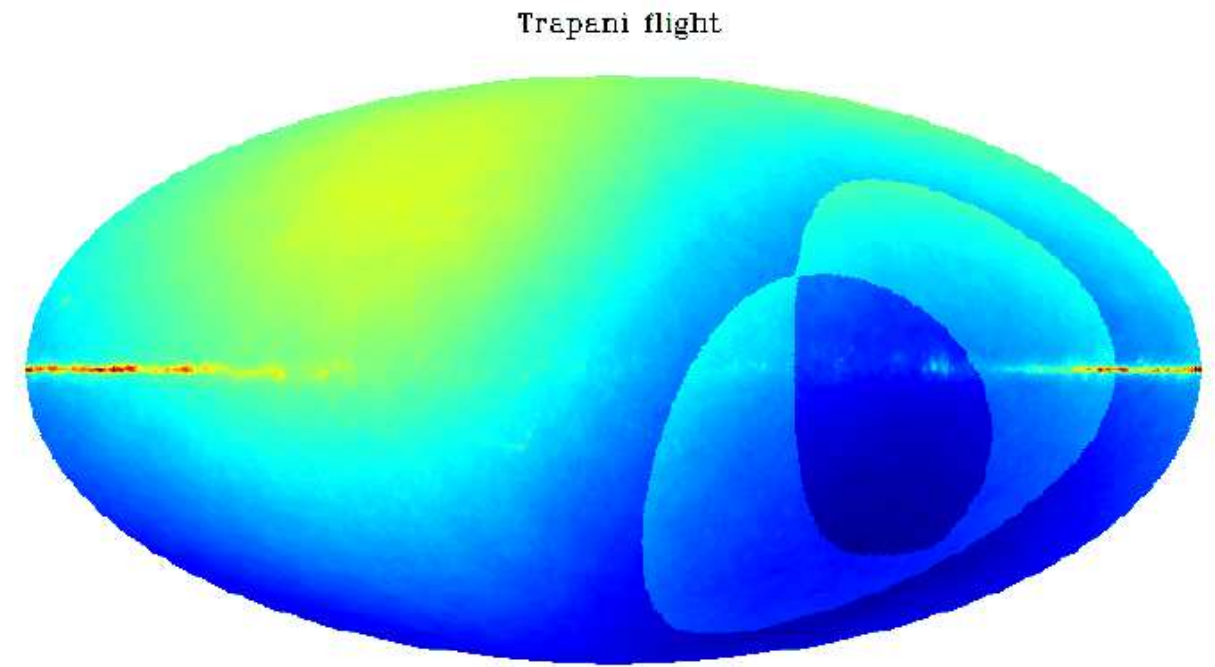

Fig. 23. All-sky Mollweide projection of the Galaxy and CMB dipole emission expected at $143 \mathrm{GHz}$, centered on the galactic anticenter. The fraction of the sky covered with the Trapani Flight during the 4 hours of dark night is shown as a brighter zone superimposed on the map. The galaxy map is taken from IRAS all-sky survey at $100 \mu \mathrm{m}$ [Schlegel et al. , 1998] and extrapolated with a dust spectrum of $17 \mathrm{~K}$ and a $n=2$ emissivity law.

reasonably consistent results (Tab. 4). Full data reduction of the Trapani flight is in progress and should be reported soon. Kiruna flight ${ }^{5}$ is being prepared using feedback from Trapani flight results. The expected sky coverage of $28 \%$ is obtained for a nominal $24 \mathrm{hr}$ flight and is shown in Fig 24.

\section{Acknowledgements}

We thank ASI (Italian Space Agency) for its continuous support during the test flight from Trapani Base. We thank Programme National de Cosmologie, Centre National d'Etudes Spatiales (CNES) and participating laboratories for their support during the instrument design and construction, and DEMIRMObservatoire de Paris for their early support. The experiment benefitted much from the early efforts by the late Richard Gispert to help us building a robust

5 A successful flight from Kiruna has just happened on the 29th, January 2001 and should be reported on soon, see http://journal .archeops.org/KirunaS1/KirunaS1.html 


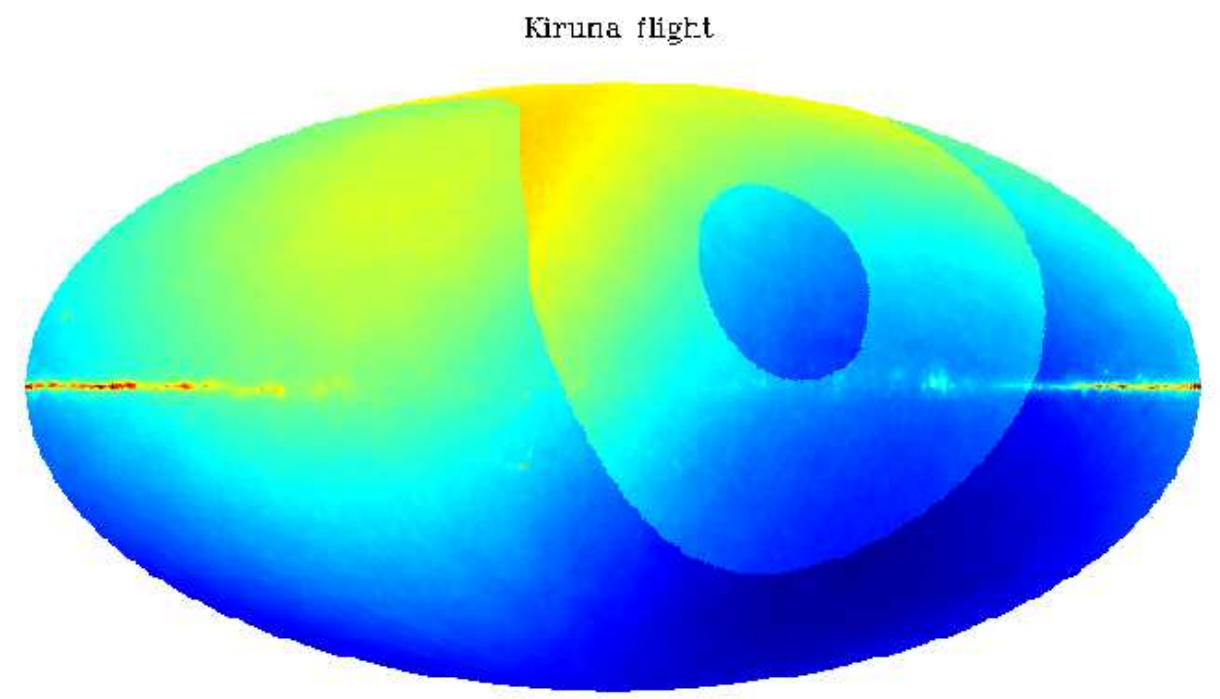

Fig. 24. Same simulated all-sky Mollweide projection as previous figure, of the Galaxy and CMB dipole emission expected at $143 \mathrm{GHz}$, centered on the galactic anticenter. The expected fraction (about 28\%) of the sky covered with the next Kiruna flight during the 24 hours is shown as a brighter zone superimposed on the map.

data reduction pipeline. We thank K. Gorski for providing us the Healpix software package.

\section{References}

[Bennett et al, 1996] Bennett C.L. et al. , 1996 Astrophys. J., 464, L1

[Benoît et al (1994a)] Benoît, A., Pujol, S., 1994a, Cryogenics, 34, 421

[Benoît et al, 1994b] Benoît, A., T. Bradshaw, C. Jewell, T. Maciaszek, A. Orlowska and S. Pujol, 1994b, Proc. 24th International Conference on Environmental Systems, 20-23 June 1994, Friedrichshafen, Germany, ICEC

[Benoît et al., 2000] Benoît, A., Zagury, F., Coron, N. et al. , 2000, A \& A Suppl. Ser., 141, 523

[Bock et al., 1996] Bock, J.J, DelCastillo, H.M., Turner, A.D., Beeman, J.W., Lange, A.E. Mauskopf, P.D., 1996, Proc. of the 30th ESLAB symp. on "Submillimetre and Far-Infrared Space Instrumentation", ESA-ESTEC 1996 
[Bouchet \& Gispert, 1998] Bouchet, F. R., \& Gispert, R., 1999, New Astron., 4, 443B

[Church et al., 1996] Church, S.E., Philhour, B., Lange, A.E., Ade, P.A.R., Maffei, B., Nartallo-Garcia, R., Dragovan, M. , 1996, Proc. of the 30th ESLAB symp. on "Submillimetre and Far-Infrared Space Instrumentation", ESA-ESTEC 1996

[Colgan et al., 2000] Colgan, R., et al. , 2000, 11th Internat. Symp. on Space Terahertz Technology, Ann Arbor, Michigan, May 2000.

[de Bernardis et al., 2000] de Bernardis P. et al. , 2000, Nature, 404, 995

[Dragone \& Hogg, 1974] Dragone, C., and Hogg, D. C., 1974, IEEE Trans. Ant. Prop., AP-22, 472

[Dragone, 1982] Dragone, C., 1982, IEEE Trans. Ant. Prop., AP-30, No. 3, 331

[Dumoulin et al. , 1993] Dumoulin, L., Berge, L., Lesueur, J., Bernas, H., Chapellier, M., 1993, Journal of Low Temperature Physics, 93, 301

[Goldin et al., 1996] Goldin, A.B. et al., 1996, ApJL, 488, L61

[Green, 1996] Green, R.E., ed. Machinery's Handbook, 25th Edition. Industrial Press, Inc., (1996), p707

[Hanany et al., 2000] Hanany S. et al., 2000, Astrophys. J. Lett., submitted, astro$\mathrm{ph} / 0005123$

[Jaffe et al., 2000] Jaffe A.H. et al., 2000, Phys. Rev. Lett., submitted, astro$\mathrm{ph} / 0007333$

[Lee et al., 1996] Lee, C., Ade, P.A.R., Haynes, C.V., 1996, Proc. of the 30th ESLAB symp. on "Submillimetre and Far-Infrared Space Instrumentation", ESA-ESTEC 1996.

[Lee et al., 2000] Lee C., et al. , 2000, to be submitted to Infrared Physics

[Maffei et al., 2000] Maffei B., Ade P.A.R., Tucker C.E., Wakui E., Wylde R.J., Murphy J.A. and Colgan R.M., 2000, Infrared and Millimetre waves, 21, 12

[Martin et al., 1996] Martin, N., Amato, M., Buchko, M., Farley, R., Frigaard, M., Generie, J., McGinnis, M., Kristensen, R., Polny, J., Dame, R., 1996, in "Space Telescopes and Instruments IV," P. Y. Bely and J. B. Breckinridge, eds., Proc. SPIE 2807 86, see also http://topweb.gsfc.nasa.gov/tophat.html

[Mauskopf et al., 1997] Mauskopf, P.D., Bock, J.J, DelCastillo, H.M., Holzapfel, W.L., Lange, A.E., 1997, Applied Optics, 36 (4), 765

[Mizuguchi, Akagawa \& Yokoi, 1978] Mizuguchi, Y., Akagawa, M., and Yokoi, H., 1978, Elect. Comm. in Japan, 61-B, No. 3, 58

[Schlegel et al. , 1998] Schlegel, David J., Finkbeiner, Douglas, P., Davis, Marc, 1998, ApJ, 500, 525 
[Sudiwala et al. , 2000a] Sudiwala, R. V., Maffei, B., Griffin, M. J., Haynes, C. V., Ade, P. A. R., Bhatia, R. S., Turner, A., Bock, J. J., Lange, A. E., Beeman, J. W., 2000a, Nucl. Instr. And Meth. A, 444, 408.

[Sudiwala et al. , 2000b] Sudiwala, R. V., 2000b, in preparation.

[Woodcraft et al. , 2000] Woodcraft et al. , 2000, in preparation. 